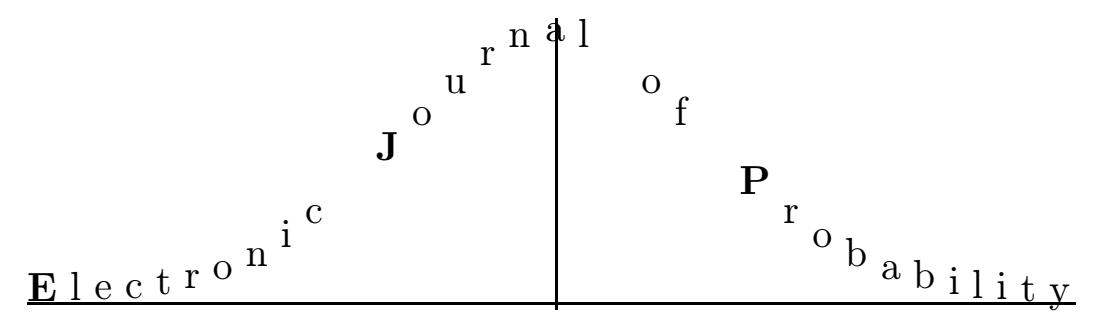

Vol. 2 (1997) Paper no. 5, pages 1-39.

Journal URL

http://www.math.washington.edu/ ejpecp/

Paper URL

http://www.math.washington.edu/ ${ }^{\sim}$ ejpecp/EjpVol2/paper5.abs.html

\title{
THE LAW OF THE ITERATED LOGARITHM FOR A TRIANGULAR ARRAY OF EMPIRICAL PROCESSES
}

\section{Miguel A. Arcones}

Department of Mathematics, University of Texas, Austin, TX 78712, arcones@math.utexas.edu

\begin{abstract}
We study the compact law of the iterated logarithm for a certain type of triangular arrays of empirical processes, appearing in statistics (M-estimators, regression, density estimation, etc). We give necessary and sufficient conditions for the law of the iterated logarithm of these processes of the type of conditions used in Ledoux and Talagrand (1991): convergence in probability, tail conditions and total boundedness of the parameter space with respect to certain pseudometric. As an application, we consider the law of the iterated logarithm for a class of density estimators. We obtain the order of the optimal window for the law of the iterated logarithm of density estimators. We also consider the compact law of the iterated logarithm for kernel density estimators when they have large deviations similar to those of a Poisson process.
\end{abstract}

Keywords: Empirical process, law of the iterated logarithm, triangular array, density estimation.

AMS subject classification: 60B12, 60F 15 .

Submitted to EJP on December 28, 1996. Final version accepted on June 22, 1997. 


\title{
The law of the logarithm iterated for a triangular array of empirical processes
}

\author{
Miguel A. Arcones ${ }^{1}$ \\ Department of Mathematics, University of Texas \\ Austin, TX 78712, arcones@math.utexas.edu
}

August 23, 1997

\begin{abstract}
We study the compact law of the iterated logarithm for a certain type of triangular arrays of empirical processes, appearing in statistics (M-estimators, regression, density estimation, etc). We give necessary and sufficient conditions for the law of the iterated logarithm of these processes of the type of conditions used in Ledoux and Talagrand (1991): convergence in probability, tail conditions and total boundedness of the parameter space with respect to certain pseudometric. As an application, we consider the law of the iterated logarithm for a class of density estimators. We obtain the order of the optimal window for the law of the iterated logarithm of density estimators. We also consider the compact law of the iterated logarithm for kernel density estimators when they have large deviations similar to those of a Poisson process.
\end{abstract}

1. Introduction. We study the law of the iterated logarithm (L.I.L.) for a certain type of triangular arrays of empirical processes. Let $\left\{X_{j}\right\}_{j=1}^{\infty}$ be a sequence of independent identically distributed random variables (i.i.d.r.v.) with values in a measurable space $(S, \mathcal{S})$. Let $\bar{T}$ be a parameter set, which has a scalar product defined for each $t \in \bar{T}$ and each $0<u \leq 1(u t \in \bar{T})$. We will assume that $u_{1}\left(u_{2} t\right)=\left(u_{1} u_{2}\right) t$, for each $u_{1}, u_{2} \in \mathbb{R}$ and each $t \in \bar{T}$. Let $g: S \times \bar{T} \rightarrow \mathbb{R}$. Here, we study the compact law of the iterated logarithm for processes of the form

$$
\left\{U_{n}(t): t \in T\right\}
$$

where $T \subset \bar{T}$,

$$
U_{n}(t):=a_{n}(2 \log \log n)^{-1 / 2} n^{-1} \sum_{j=1}^{n}\left(g\left(X_{j}, b_{n} t\right)-E\left[g\left(X_{j}, b_{n} t\right)\right]\right)
$$

and $\left\{a_{n}\right\}_{n=1}^{\infty}$ and $\left\{b_{n}\right\}_{n=1}^{\infty}$ are sequences of positive numbers with $0<b_{n} \leq 1$. This problem has been considered in Arcones (1994) when $T=\bar{T}$ and the functions are uniformly bounded. An

\footnotetext{
${ }^{1}$ Research partially supported by NSF Grant DMS-93-02583 and carried out at the departments of Mathematics of the University of Utah and of the University of Texas,
} 
extension of this work is needed to get the results in Arcones (1996a, 1996b). Here, we obtain necessary and sufficient conditions for the compact L.I.L. for $\left\{U_{n}(t): t \in T\right\}$. This type of processes appears often in statistics, such as in the study of M-estimators (Arcones 1994, 1996a), in regression estimation (see Härdle, 1984; Hall, 1991; and Arcones, 1996b), in density estimation (see Parzen, 1962; Hall, 1981; Härdle, 1984; Deheuvels and Mason, 1994; and Section 4 below) and in estimation for stochastic processes (Prakasa Rao, 1983). As a particular case, we get the L.I.L. of processes of the form

$$
\left\{V_{n}(t): t \in T\right\}
$$

where

$$
V_{n}(t):=\left(n b_{n}^{d} 2 \log \log n\right)^{-1 / 2} \sum_{j=1}^{n}\left(K_{t}\left(b_{n}^{-1}\left(x_{0}-X_{j}\right)\right)-E\left[K_{t}\left(b_{n}^{-1}\left(x_{0}-X_{j}\right)\right)\right]\right),
$$

$\left\{X_{j}\right\}_{j=1}^{\infty}$ is a sequence of i.i.d.r.v.'s with values in $\mathbb{R}^{d}, x_{0} \in \mathbb{R}^{d}$ and $\left\{K_{t}: t \in T\right\}$ is a class of functions in $\mathbb{R}^{d}$. We apply this L.I.L. to density estimation.

In Section 2, some general facts on necessary and sufficient conditions for the L.I.L. of the process $\left\{U_{n}(t): t \in T\right\}$ are presented. In particular, we show the a.s. asymptotic equicontinuity of $\left\{U_{n}(t): t \in T\right\}$ under certain conditions.

In Section 3, we give, under some regularity conditions, necessary and sufficient conditions for the compact L.I.L. of $\left\{U_{n}(t): t \in T\right\}$ when the limit set is related with a Gaussian process, similar to the conditions of Ledoux and Talagrand (1988) for random variables with values in a Banach space: convergence in probability, tail conditions and total boundedness of the parameter space with respect to certain pseudometric. The key ingredient in the proofs is the Talagrand isoperimetric inequality for a sum of independent Banach-valued random variables (Theorem 6.17 in Ledoux and Talagrand, 1991). We also consider sufficient conditions for the compact LIL for the processes in (1.1) and (1.2) when the class of functions is either a VC subgraph class or satisfies certain bracketing conditions. In the considered situations in this section the limit set of the sequence (1.1) is the unit of the reproducing kernel Hilbert space of the Gaussian process with the limit covariance of $\left\{a_{n} n^{-1} \sum_{j=1}^{n}\left(g\left(X_{j}, b_{n} t\right)-E\left[g\left(X_{j}, b_{n} t\right)\right]\right): t \in T\right\}$.

In Section 4, we consider the L.I.L. for the processes in (1.2) whose limit set is related with a Gaussian process. We apply these results to the L.I.L. for density estimators. We obtain the rate of the optimal window in the LIL of density estimators. The conditions that we impose differ from those of the literature. From example, Deheuvels and Mason (1994, Theorem C) imposed that the density to be twice continuous differentiable in a neighborhood of $x_{0}$, we only will assume first differentiability with continuity.

In Section 5, we consider the L.I.L. for the processes in (1.2) whose limit set is related with a Poisson process. In this section, we extend the results in Deheuvels and Mason (1990, 1991, 1995)

We use the notation from empirical processes. Given a measurable function $g$ on $(S, \mathcal{S})$ and a 
probability measure $\mu$ on $S, \mu g:=\int g d \mu$, in particular,

$$
P_{n} g:=n^{-1} \sum_{i=1}^{n} g\left(X_{i}\right) \text { and } P g:=E[g(X)],
$$

where $X$ is a copy of $X_{1} \cdot l_{\infty}(T)$ will denote the Banach space consisting of the bounded functions on $T$ with the supremum norm. As usual, we need to assume some measurability conditions. Let $\Omega:=S^{\mathbb{N}} \times[0,1]^{\mathbb{N}}$, let $\mathcal{A}$ be the product $\sigma$-algebra on $\Omega$ when $S$ is endowed with the $\sigma$-algebra $\mathcal{A}$ and $[0,1]$ is endowed with the Borel $\sigma$-algebra. In $(\Omega, \mathcal{A})$, we define the probability function $\operatorname{Pr}$ as the product of the probability function $P$ in each $S$ factor and the Lebesgue measure defined in each $[0,1]$ factor. The r.v.'s $X_{i}$ is the function defined in $\Omega=S^{\mathbb{N}} \times[0,1]^{\mathbb{N}}$ which takes the value of the $i$-th coordinate of $S^{I N}$. $\left\{\epsilon_{i}\right\}_{i=1}^{n}$ will a Rademacher sequence independent r.v.'s, independent of the sequence $\left\{X_{i}\right\}$ and defined in the same probability space $(\Omega, \mathcal{A}, \operatorname{Pr})$. We also will assume that the class of functions $\{g(x, u t): 0<u \leq 1, t \in T\}$ is an image admissible Suslin class of functions in the sense of Dudley (1984, page 101). Sometimes, it will be enough to assume that $\left\{g(x, u t): 1-\delta_{0} \leq u \leq 1, t \in T\right\}$ is an image admissible Suslin class of functions for some $0<\delta_{0}<1$.

We will denote to a finite partition $\pi$ of $T$ to a map $\pi: T \rightarrow T$ such that $\pi(\pi(t))=\pi(t)$, for each $t \in T$, and the cardinality of $\{\pi(t): t \in T\}$ is finite.

2. Some general facts on the LIL for triangular arrays of empirical processes. In this section, we present some general facts on the L.I.L. of $\left\{U_{n}(t): t \in T\right\}$. First we recall some sufficient conditions for the L.I.L. of stochastic processes (see e.g. Arcones and Giné, 1995):

LEMMA 2.1. Let $\left\{U_{n}(t): t \in T\right\}, n \geq 1$, be any sequence of stochastic processes, suppose that the following conditions are satisfied:

(i) There exists a set $C \subset l_{\infty}(T)$ such that for each $t_{1}, \ldots, t_{m} \in T$, with probability one, the sequence $\left\{\left(U_{n}\left(t_{1}\right), \ldots, U_{n}\left(t_{m}\right)\right)\right\}_{n=1}^{\infty}$ is relatively compact in $\mathbb{R}^{m}$ and its limit set is

$$
\left\{\left(x\left(t_{1}\right), \ldots, x\left(t_{m}\right)\right): x \in C\right\} .
$$

(ii) There exists a pseudometric $\rho$ in $T$ such that $(T, \rho)$ is totally bounded.

(iii) $\lim _{\delta \rightarrow 0} \lim \sup _{n \rightarrow \infty} \sup _{\rho(s, t) \leq \delta}\left|U_{n}(s)-U_{n}(t)\right|=0$ a.s.

Then, with probability one, $\left\{U_{n}(t): t \in T\right\}$ is relatively compact in $l_{\infty}(T)$ and its limit set is C.

We have the following necessary conditions for the the compact L.I.L. of the processes in (1.1):

LEMMA 2.2. With the former notation, assume that:

(i) For each $n, 0<b_{n} \leq 1$.

(ii) There exists compact set $C \in l_{\infty}(T)$ such that, with probability one $\left\{U_{n}(t): t \in T\right\}$ is relatively compact in $l_{\infty}(T)$ and its limit set is $C$.

(iii) For each $t \in T, U_{n}(t) \stackrel{\operatorname{Pr}}{\longrightarrow} 0$.

(iv) $a_{n} a_{n-1}^{-1}=O(1)$. 
Then,

(a) $(T, \rho)$ is totally bounded, where $\rho(s, t)=\sup _{x \in C}|x(s)-x(t)|$.

(b) $\lim _{\delta \rightarrow 0} \lim \sup _{n \rightarrow \infty} \sup _{\rho(s, t) \leq \delta}\left|U_{n}(s)-U_{n}(t)\right|=0$ a.s.

(c) $\sup _{t \in T}\left|U_{n}(t)\right| \stackrel{\mathrm{Pr}}{\longrightarrow} 0$.

Moreover, if $T=\bar{T}$ and $\left\{b_{n}\right\}$ is non-increasing,

(d) There exists a finite constant $c$ such that

$$
\sum_{n=1}^{\infty} \operatorname{Pr}\left\{G\left(X, b_{n}\right) \geq c n(\log \log n)^{1 / 2} a_{n}^{-1}\right\}<\infty
$$

where

$$
G\left(x, b_{n}\right):=\sup _{t \in T}\left|g\left(x, b_{n} t\right)-E\left[g\left(X, b_{n} t\right)\right]\right| .
$$

ProOF. It is well known that in this situation: $(T, \rho)$ is totally bounded and $C$ is contained in the set of bounded and $\rho$-uniformly continuous functions on $T$ (see Arcones and Giné, 1995) . This implies conditions (a) and (b). Condition (b) implies that

$$
\lim _{\delta \rightarrow 0} \limsup _{n \rightarrow \infty} \operatorname{Pr}\left\{\sup _{\rho(s, t) \leq \delta}\left|U_{n}(s)-U_{n}(t)\right| \geq \eta\right\}=0
$$

for each $\eta>0$. This fact and conditions (iii) and (a) imply (c). We have that

$$
\limsup _{n \rightarrow \infty} \sup _{t \in T}\left|U_{n}(t)\right|=\sup _{t \in T} \sup _{x \in C}|x(t)| \text { a.s. }
$$

and

$$
\begin{gathered}
\limsup _{n \rightarrow \infty} a_{n}(2 \log \log n)^{-1 / 2} n^{-1} \sup _{t \in T}\left|\sum_{j=1}^{n-1}\left(g\left(X_{j}, b_{n} t\right)-E\left[g\left(X_{j}, b_{n} t\right)\right]\right)\right| \\
\leq \limsup _{n \rightarrow \infty}\left(a_{n} a_{n-1}^{-1}\right) a_{n-1}(2 \log \log (n-1))^{-1 / 2}(n-1)^{-1} \sup _{t \in T}\left|\sum_{j=1}^{n-1}\left(g\left(X_{j}, b_{n-1} t\right)-E\left[g\left(X_{j}, b_{n-1} t\right)\right]\right)\right| \\
\leq \limsup _{n \rightarrow \infty}\left(a_{n} a_{n-1}^{-1}\right) \sup _{t \in T} \sup _{x \in C}|x(t)| \text { a.s. }
\end{gathered}
$$

So,

$$
\limsup _{n \rightarrow \infty} a_{n}(2 \log \log n)^{-1 / 2} n^{-1} G\left(X_{n}, b_{n}\right) \leq c \text { a.s. }
$$

for some finite constant $c$. From this and the lemma of Borel-Cantelli, (d) follows.

The problem is to show conditions (i) and (iii) in Lemma 2.1. In order to check these conditions, we will use the following exponential inequality for empirical processes (Talagrand isoperimetric inequality):

Theorem 2.3. (Theorem 6.17 in Ledoux and Talagrand, 1991). Let $\left\{X_{1}(t)\right\}_{t \in T}, \ldots,\left\{X_{n}(t)\right\}_{t \in T}$ be independent random processes indexed by $T$ and let $\left\{\epsilon_{i}\right\}_{i=1}^{n}$ be a 
Rademacher sequence independent of the last processes. Then, for any integers $k \geq q$ and any real numbers $s, t>0$

$$
\begin{gathered}
\operatorname{Pr}\left\{\sup _{t \in T}\left|\sum_{i=1}^{n} \epsilon_{i} X_{i}(t)\right| \geq 8 q M+2 s+t\right\} \\
\leq\left(\frac{K_{0}}{q}\right)^{k}+\operatorname{Pr}\left\{\sum_{i=1}^{k} Y_{i}^{*} \geq s\right\}+2 \exp \left(-\frac{t^{2}}{128 q m^{2}}\right),
\end{gathered}
$$

where $K_{0}$ is a universal constant, $M:=E\left[\sup _{t \in T}\left|\sum_{i=1}^{n} \epsilon_{i} X_{i}(t) I_{\left\|X_{i}\right\| \leq s k^{-1}}\right|\right],\left\|X_{i}\right\|:=\sup _{t \in T}\left|X_{i}(t)\right|$,

$$
m:=E\left[\sup _{t \in T}\left(\sum_{j=1}^{n} X_{i}^{2}(t) I_{\left\|X_{i}\right\| \leq s / k}\right)^{1 / 2}\right]
$$

and $\left\{Y_{i}^{*}\right\}_{i=1}^{n}$ denotes the non-increasing rearrangement of $\sup _{t}\left|X_{i}(t)\right|, 1 \leq i \leq n$.

We must notice that

$$
m^{2} \leq \sup _{t \in T} \sum_{i=1}^{n} E\left[X_{i}^{2}(t) I_{\left\|X_{i}\right\| \leq s / k}\right]+8 M s k^{-1}
$$

(see Equation (6.18) in Ledoux and Talagrand, 1991).

The following theorem gives the a.s. asymptotic equicontinuity of the process $\left\{U_{n}(t): t \in T\right\}$.

THEOREM 2.4. With the above notation, suppose that the following conditions are satisfied:

(i) $\left\{b_{n}\right\}$ is a non-increasing sequence of numbers from the interval $(0,1]$.

(ii) $\lim _{\gamma \rightarrow 1+} \lim \sup _{n \rightarrow \infty} \sup _{m: n \leq m \leq \gamma n}\left|a_{n}^{-1} a_{m}-1\right|=0$

and $\lim _{\gamma \rightarrow 1+} \lim \sup _{n \rightarrow \infty} \sup _{m: n \leq m \leq \gamma n}\left|b_{n}^{-1} b_{m}-1\right|=0$.

(iii) There are constants $r_{1}, r_{2}>0,1>\delta_{0}>0$, such that

$$
\limsup _{\gamma \rightarrow 1+} \sum_{j=2}^{\infty} \sup _{r_{1}(\log j)^{-1} \leq r \leq r_{2}} e^{-r_{1} r^{-1}} \gamma^{j} \operatorname{Pr}\left\{G_{\delta_{0}}\left(X, b_{\left[\gamma^{j}\right]}\right) \geq r \gamma^{j}(\log j)^{1 / 2} a_{\left[\gamma^{j}\right]}^{-1}\right\}<\infty,
$$

where

$$
G_{\delta_{0}}\left(x, b_{n}\right):=\sup _{t \in T} \sup _{1-\delta_{0} \leq u \leq 1}\left|g\left(x, u b_{n} t\right)-E\left[g\left(X, u b_{n} t\right)\right]\right| .
$$

(iv) For each $\eta>0$,

$$
\lim _{\delta \rightarrow 0} \limsup _{n \rightarrow \infty} \operatorname{Pr}\left\{\sup _{1-\delta \leq u \leq 1} \sup _{t \in T}\left|U_{n}(u t)\right| \geq \eta\right\}=0 .
$$

(v) For each $\eta>0$, there exists a $\delta>0$ such that

$$
\limsup _{\gamma \rightarrow 1+} \sum_{j=2}^{\infty} \exp \left(-\frac{\eta \log j}{\sup _{\substack{s, t \in T \\ \rho(s, t) \leq \delta}} s_{\left[\gamma^{j}\right]}^{2}(s, t)}\right)<\infty
$$


and

$$
\limsup _{\gamma \rightarrow 1+} \sum_{j=2}^{\infty} \exp \left(-\frac{\eta \log j}{\sup _{1-\delta \leq u \leq 1} \sup _{t \in T} s_{\left[\gamma^{j}\right]}^{2}(u t, t)}\right)<\infty,
$$

where $s_{n}^{2}(s, t)=a_{n}^{2} n^{-1} \operatorname{Var}\left(g\left(X, b_{n} s\right)-g\left(X, b_{n} t\right)\right)$.

Then,

$$
\lim _{\delta \rightarrow 0} \limsup _{n \rightarrow \infty} \sup _{\substack{s, t \in T \\ \rho(s, t) \leq \delta}}\left|U_{n}(s)-U_{n}(t)\right|=0 \quad \text { a.s. }
$$

Proof. Without loss of generality, we may assume that $E[g(X, t)]=0$ for each $t \in T$. Given $2^{-2}>\eta>0$, take $\delta>0$ and $\gamma$ (in this order), such that

$$
\begin{gathered}
0<\delta \leq \delta_{0} ; \sum_{j=2}^{\infty} \exp \left(-\frac{\eta^{2} \log j}{\sup _{\rho(s, t) \leq \delta} s_{\left[\gamma^{j}\right]}^{2}(s, t)}\right)<\infty ; \\
\sum_{j=2}^{\infty} \exp \left(-\frac{\eta^{2} \log j}{\sup _{1-\delta \leq u \leq 1} \sup _{t \in T} s_{\left[\gamma^{j}\right]}^{2}(u t, t)}\right)<\infty ; 1<\gamma<1+\eta ; \\
\sum_{j=2}^{\infty} \sup _{r_{1}(\log j)^{-1} \leq r \leq r_{2}} e^{-r_{1} r^{-1}} \gamma^{j} \operatorname{Pr}\left\{G_{\delta_{0}}\left(X, b_{\left[\gamma^{j}\right]}\right) \geq r \gamma^{j}(\log j)^{1 / 2} a_{\left[\gamma^{j}\right]}^{-1}\right\}<\infty ; \\
\limsup _{n \rightarrow \infty} \sup _{m: n \leq m \leq \gamma n}\left|b_{n}^{-1} b_{m}-1\right|<1-\delta \text { and } \\
\limsup _{n \rightarrow \infty} \sup _{m: n \leq m \leq \gamma n}\left|(2 \log \log n)^{-1 / 2} a_{n} n^{-1}(2 \log \log m)^{1 / 2} a_{m}^{-1} m-1\right|<\eta .
\end{gathered}
$$

Let $n_{j}=\left[\gamma^{j}\right]$. We claim that by the Talagrand isoperimetric inequality

$$
\limsup _{j \rightarrow \infty} \sup _{\substack{t, s \in T \\ \rho(t, s) \leq \delta}}\left|U_{n_{j}}(t)-U_{n_{j}}(s)\right| \leq 2^{8} K_{0}^{1 / 2} \eta^{1 / 2}+2^{5}\left(r_{1}+1\right)\left(\log \eta^{-1}\right)^{-1} \quad \text { a.s. },
$$

where $K_{0}$ is the constant in Theorem 2.3. By condition (v),

$$
\sup _{\substack{s, t \in T \\ \rho(s, t) \leq \delta}}\left(\log \log \gamma^{j}\right)^{-1} s_{\left[\gamma^{j}\right]}^{2}(s, t) \rightarrow 0
$$

So, by symmetrization (see for example Lemma 1.2.1 in Giné and Zinn, 1986),

$$
\begin{gathered}
\operatorname{Pr}\left\{\sup _{\rho(s, t) \leq \delta}(2 \log j)^{-1 / 2} a_{n_{j}}\left|\left(P_{n_{j}}-P\right)\left(g\left(\cdot, b_{n_{j}} s\right)-g\left(\cdot, b_{n_{j}} t\right)\right)\right|\right. \\
\left.\geq 2^{8} K_{0}^{1 / 2} \eta^{1 / 2}+32\left(r_{1}+1\right)\left(\log \eta^{-1}\right)^{-1}\right\} \\
\leq 4 \operatorname{Pr}\left\{\sup _{\rho(s, t) \leq \delta}(\log j)^{-1 / 2} a_{n_{j}} n_{j}^{-1}\left|\sum_{i=1}^{n_{j}} \epsilon_{i}\left(g\left(X_{i}, b_{n_{j}} s\right)-g\left(X_{i}, b_{n_{j}} t\right)\right)\right|\right. \\
\left.\geq 65 K_{0}^{1 / 2} \eta^{1 / 2}+16\left(r_{1}+1\right)\left(\log \eta^{-1}\right)^{-1}\right\},
\end{gathered}
$$


for $j$ large, where $\left\{\epsilon_{i}\right\}$ is a sequence of i.i.d. Rademacher r.v.'s independent of the sequence $\left\{X_{i}\right\}$. Now, we claim that by Theorem 2.3 last expression is bounded by

$$
8 j^{-2}+16\left(\eta^{-r}+2\right) \delta_{j}+8 \exp \left(-\frac{\eta^{2} \log j}{\sup _{\rho(s, t) \leq \delta} s_{n_{j}}^{2}(s, t)}\right), \text { for } j \text { large, }
$$

where

$$
\delta_{j}:=\sup _{r_{1}(\log j)^{-1} \leq t \leq r_{2}} e^{-r_{1} t^{-1}} \gamma^{j} \operatorname{Pr}\left\{G\left(X, b_{\left[\gamma^{j}\right]}\right) \geq t \gamma^{j}(\log j)^{1 / 2} a_{\left[\gamma^{j}\right]}^{-1}\right\} .
$$

We consider two cases. If

$$
\delta_{j} \leq j^{-3}
$$

we take

$$
\begin{gathered}
q=K_{0} \eta^{-1}, k=2(\log j)\left(\log \eta^{-1}\right)^{-1}, \\
t=2^{6} K_{0}^{1 / 2} \eta^{1 / 2}(\log j)^{1 / 2} \text { and } s=8\left(1+r_{1}\right)\left(\log \eta^{-1}\right)^{-1}(\log j)^{1 / 2} .
\end{gathered}
$$

We have that $\left(K_{0} / q\right)^{k}=j^{-2}$ and

$$
\sum_{i=1}^{k} Y_{n_{j}, i}^{*} \leq 4(\log j)\left(\log \eta^{-1}\right)^{-1} a_{n_{j}} n_{j}^{-1} \max _{1 \leq i \leq n_{j}} G\left(X_{i}, b_{n_{j}}\right)
$$

where $\left\{Y_{n_{j}, i}^{*}\right\}_{i=1}^{n_{j}}$ is a non-increasing rearrangement of

$$
\sup _{\rho(s, t) \leq \delta} a_{n_{j}} n_{j}^{-1}\left|g\left(X_{i}, b_{n_{j}} s\right)-g\left(X_{i}, b_{n_{j}} t\right)\right|, 1 \leq i \leq n_{j} .
$$

From this estimation and (2.7),

$$
\operatorname{Pr}\left\{\sum_{i=1}^{k} Y_{n_{j}, i}^{*} \geq s\right\} \leq n_{j} \operatorname{Pr}\left\{G\left(X, b_{n_{j}}\right) \geq 2\left(r_{1}+1\right) n_{j} a_{n_{j}}^{-1}(\log j)^{-1 / 2}\right\} \leq j^{-2} .
$$

We have that by the Hoffmann-Jørgensen inequality (see for example Proposition 6.8 in Ledoux and Talagrand, 1991)

$$
E\left[\sup _{t \in T} a_{n_{j}}(\log j)^{-1 / 2} n_{j}^{-1}\left|\sum_{i=1}^{n_{j}} \epsilon_{i} g\left(X_{i}, b_{n_{j}} t\right) I_{G\left(X_{i}, b_{n_{j}}\right) \leq c n_{j} a_{n_{j}}^{-1}(\log j)^{-1 / 2}}\right|\right] \rightarrow 0
$$

for any $c<\infty$. So,

$$
\begin{gathered}
a_{n_{j}}^{2} n_{j}^{-1} \sup _{\rho(s, t) \leq \delta} E\left[\left(g\left(X, b_{n_{j}} s\right)-g\left(X_{i}, b_{n_{j}} t\right)\right)^{2}\right] \\
+(8 s / k) E\left[\sup _{\rho(s, t) \leq \delta} a_{n_{j}} n_{j}^{-1}\left|\sum_{i=1}^{n_{j}} \epsilon_{i}\left(g\left(X_{i}, b_{n_{j}} s\right)-g\left(X_{i}, b_{n_{j}} t\right)\right)\right|\right] \\
\leq \sup _{\rho(s, t) \leq \delta} s_{n_{j}}^{2}(s, t)+o(1), \text { for } j \text { large. }
\end{gathered}
$$

From the former estimations, it follows that (2.5) is bounded by (2.6). 
If (2.7) does not hold, we take $q, t$ and $s$ as before and $k=\left(\log \delta_{j}^{-1}\right)\left(\log \eta^{-1}\right)^{-1}$. Using that

$$
\sum_{i=1}^{k} Y_{n_{j}, i}^{*} \leq l Y_{n_{j}, 1}^{*}+k Y_{n_{j}, l}^{*}
$$

where $l:=\left[4+4 r_{1}\right]$, we get that

$$
\operatorname{Pr}\left\{\sum_{i=1}^{k} Y_{n_{j}, i}^{*} \geq s\right\} \leq \operatorname{Pr}\left\{Y_{n_{j}, 1}^{*} \geq 2^{-1} l^{-1} s\right\}+\operatorname{Pr}\left\{Y_{n_{j}, l}^{*} \geq 2^{-1} k^{-1} s\right\} \leq \delta_{j}\left(\eta^{-r_{1}}+\eta^{-2 r_{1}}\right) .
$$

Again by the Hoffmann-Jørgensen inequality

$$
E\left[\sup _{t \in T} a_{n_{j}}(\log j)^{-1 / 2} n_{j}^{-1}\left|\sum_{i=1}^{n_{j}} \epsilon_{i} g\left(X_{i}, b_{n_{j}} t\right) I_{G\left(X_{i}, b_{n_{j}}\right) \leq c n_{j} a_{n_{j}}^{-1}(\log j)^{1 / 2}\left(\log \delta_{j}^{-1}\right)^{-1}}\right|\right] \rightarrow 0,
$$

for any $c<\infty$. So,

$$
\begin{gathered}
a_{n_{j}}^{2} n_{j}^{-1} \sup _{\rho(s, t) \leq \delta} E\left[\left(g\left(X, b_{n_{j}} s\right)-g\left(X_{i}, b_{n_{j}} t\right)\right)^{2}\right] \\
+\left(2^{4} s / k\right) E\left[\sup _{t \in T} a_{n_{j}}(\log j)^{-1 / 2} n_{j}^{-1} \mid \sum_{i=1}^{n_{j}} \epsilon_{i} g\left(X_{i}, b_{n_{j}} t\right) I_{\left.G\left(X_{i}, b_{n_{j}}\right) \leq c n_{j} a_{n_{j}}^{-1}(\log j)^{1 / 2}\left(\log \delta_{j}^{-1}\right)^{-1} \mid\right]}\right. \\
\leq \sup _{\rho(s, t) \leq \delta} s_{n_{j}}^{2}(s, t)+o\left((\log j)\left(\log \delta_{j}^{-1}\right)^{-1}\right) .
\end{gathered}
$$

Hence, (2.5) is bounded by (2.6).

Next, we will prove that

$$
\begin{gathered}
\limsup _{j \rightarrow \infty} \sup _{\rho(s, t) \leq \delta} \sup _{n_{j}<n \leq n_{j+1}}\left|U_{n}(t)-U_{n_{j}}(t)-U_{n}(s)+U_{n_{j}}(s)\right| \\
\leq 2^{11} K_{0}^{1 / 2} \eta^{1 / 2}+2^{8}\left(r_{1}+1\right)\left(\log \eta^{-1}\right)^{-1} .
\end{gathered}
$$

We have that, for $n_{j}<n$,

$$
\begin{gathered}
(2 \log \log n)^{-1 / 2} a_{n}\left(P_{n}-P\right) g\left(\cdot, b_{n} t\right)-\left(2 \log \log n_{j}\right)^{-1 / 2} a_{n_{j}}\left(P_{n_{j}}-P\right) g\left(\cdot, b_{n_{j}} t\right) \\
=\left((2 \log \log n)^{-1 / 2} a_{n} n^{-1}-\left(2 \log \log n_{j}\right)^{-1 / 2} a_{n_{j}} n_{j}^{-1}\right) \sum_{i=1}^{n_{j}} g\left(X_{i}, b_{n_{j}} t\right) \\
+(2 \log \log n)^{-1 / 2} a_{n} n^{-1} \sum_{i=n_{j}+1}^{n} g\left(X_{i}, b_{n_{j}} t\right) \\
+(2 \log \log n)^{-1 / 2} a_{n} n^{-1} \sum_{i=1}^{n}\left(g\left(X_{i}, b_{n} t\right)-g\left(X_{i}, b_{n_{j}} t\right)\right) \\
=: I_{n}(t)+I I_{n}(t)+I I I_{n}(t) .
\end{gathered}
$$

By (2.3), for $n$ large,

$$
\sup _{n_{j}<n \leq n_{j+1}} \sup _{\rho(s, t) \leq \delta}\left|I_{n}(t)-I_{n}(s)\right|
$$




$$
\leq \eta \sup _{\rho(s, t) \leq \delta}\left(2 \log \log n_{j}\right)^{-1 / 2} a_{n_{j}} n_{j}^{-1}\left|\sum_{i=1}^{n_{j}} g\left(X_{i}, b_{n_{j}} t\right)\right|
$$

Therefore,

$$
\limsup _{j \rightarrow \infty} \sup _{n_{j}<n \leq n_{j+1}} \sup _{\rho(s, t) \leq \delta}\left|I_{n}(s)-I_{n}(t)\right| \leq 2^{9} K_{0}^{1 / 2} \eta^{1 / 2}+2^{6}\left(r_{1}+1\right)\left(\log \eta^{-1}\right)^{-1} \text { a.s. }
$$

By symmetrization and the Lévy inequality,

$$
\begin{gathered}
\operatorname{Pr}\left\{\sup _{n_{j}<n \leq n_{j+1}} \sup _{\rho(s, t) \leq \delta}\left|I I_{n}(s)-I I_{n}(t)\right| \geq 2^{9} K_{0}^{1 / 2} \eta^{1 / 2}+2^{6}\left(r_{1}+1\right)\left(\log \eta^{-1}\right)^{-1}\right\} \\
\leq 8 \operatorname{Pr}\left\{\sup _{\rho(s, t) \leq \delta}\left(2 \log \log n_{j}\right)^{-1 / 2} a_{n_{j}} n_{j}^{-1}\left|\sum_{i=n_{j}+1}^{n_{j+1}} \epsilon_{i}\left(g\left(X_{i}, b_{n_{j}} s\right)-g\left(X_{i}, b_{n_{j}} t\right)\right)\right|\right. \\
\left.\geq 65 K_{0}^{1 / 2} \eta^{1 / 2}+16\left(r_{1}+1\right)\left(\log \eta^{-1}\right)^{-1}\right\} .
\end{gathered}
$$

So, from this and the estimation of (2.5),

$$
\lim _{j \rightarrow \infty} \sup _{n_{j}<n \leq n_{j+1}} \sup _{\rho(s, t) \leq \delta}\left|I I_{n}(s)-I I_{n}(t)\right| \leq 2^{9} K_{0}^{1 / 2} \eta^{1 / 2}+2^{6}\left(r_{1}+1\right)\left(\log \eta^{-1}\right)^{-1} \text { a.s. }
$$

By (2.3), $\sup _{n_{j}<n \leq n_{j+1}}\left|b_{n_{j}}^{-1} b_{n}-1\right| \leq 1-\delta$, for $j$ large. So,

$$
\begin{gathered}
\sup _{n_{j}<n \leq n_{j+1}} \sup _{t \in T}\left|I I I_{n}(t)\right| \\
\leq \eta \sup _{1-\delta \leq u \leq 1} \sup _{t \in T} \sup _{n_{j}<n \leq n_{j+1}}\left(2 \log \log n_{j}\right)^{-1 / 2} a_{n_{j}} n_{j}^{-1}\left|\sum_{i=1}^{n}\left(g\left(X_{i}, u b_{n_{j}} t\right)-g\left(X_{i}, b_{n_{j}} t\right)\right)\right| .
\end{gathered}
$$

By the argument leading to (2.4)

$$
\begin{gathered}
\limsup _{j \rightarrow \infty} \sup _{1-\delta \leq u \leq 1} \sup _{t \in T} \sup _{n_{j}<n \leq n_{j+1}}\left(2 \log \log n_{j}\right)^{-1 / 2} a_{n_{j}} n_{j}^{-1}\left|\sum_{i=1}^{n}\left(g\left(X_{i}, u b_{n_{j}} t\right)-g\left(X_{i}, b_{n_{j}} t\right)\right)\right| \\
\leq 2^{8} K_{0}^{1 / 2} \eta^{1 / 2}+2^{5}\left(r_{1}+1\right)\left(\log \eta^{-1}\right)^{-1} \quad \text { a.s. }
\end{gathered}
$$

Hence,

$$
\limsup _{j \rightarrow \infty} \sup _{n_{j}<n \leq n_{j+1}} \sup _{t \in T}\left|I I I_{n}(t)\right| \leq 2^{9} K_{0}^{1 / 2} \eta^{1 / 2}+2^{6}\left(r_{1}+1\right)\left(\log \eta^{-1}\right)^{-1} \text { a.s. }
$$

(2.8) follows, from (2.9)-(2.12). (2.4) and (2.8) imply (2.2)

REMARKs 2.5. Conditions (i) and (ii) in Theorem 2.4 are easy to check. We must observe that condition (iii) in Theorem 2.4 is similar to condition (7.7) in Ledoux and Talagrand (1991). In order to check this condition, it suffices to prove any of the following conditions:

(c.1) There exist positive constants $r_{1}, r_{2}$ and $p$ such that

$$
\lim _{\gamma \rightarrow 1+} \sum_{j=2}^{\infty} \sup _{r_{1}(\log j)^{-1} \leq r \leq r_{2}} r^{p} \gamma^{j} \operatorname{Pr}\left\{G_{\delta_{0}}\left(X, b_{\left[\gamma^{j}\right]}\right) \geq r \gamma^{j}(\log j)^{1 / 2} a_{\left[\gamma^{j}\right]}^{-1}\right\}<\infty .
$$


(c.2) There exists a constant $r_{1}>0$ such that

$$
a_{n}(\log \log n)^{1 / 2} n^{-1} G_{\delta_{0}}\left(X, b_{n}\right) \leq r_{1} \text { a.s. }
$$

for each $n$ large enough.

(c.3) There exist positive constants $r_{1}, r_{2}$ and $p$ such that

$$
\lim _{\gamma \rightarrow 1+} \sum_{j=2}^{\infty} \gamma^{j} \operatorname{Pr}\left\{G_{\delta_{0}}\left(X, b_{\left[\gamma^{j}\right]}\right) \geq r_{2} \gamma^{j}(\log j)^{1 / 2} a_{\left[\gamma^{j}\right]}^{-1}\right\}<\infty
$$

and

$$
\lim _{\gamma \rightarrow 1+} \sum_{j=2}^{\infty} a_{\left[\gamma^{j}\right]}^{p} \gamma^{j(-p+1)}(\log j)^{p / 2} E\left[G_{\delta_{0}}^{p}\left(X, b_{\left[\gamma^{j}\right]}\right) I_{\left.r_{1} \gamma^{j}(\log j)^{-1 / 2} a_{\left[\gamma^{j}\right]}^{-1} \leq G_{\delta_{0}}\left(X, b_{\left[\gamma^{j}\right]}\right) \leq r_{2} \gamma^{j}(\log j)^{1 / 2} a_{\left[\gamma^{j}\right]}^{-1}\right]}\right]<\infty .
$$

(c.4) There exists an integer $n_{0}$ such that $E\left[H^{2}(X)(\log \log (H(X)+e))^{-1}\right]<\infty$, where

$$
H(x):=\sup _{n \geq n_{0}} a_{n} n^{-1 / 2} G_{\delta_{0}}\left(x, b_{n}\right) .
$$

(c.1) implies from condition (iii) because $e^{r_{1} r^{-1}} r^{p}=0(1)$, uniformly on a bounded interval. (c.2) follows immediately from (c.1). It is easy to see that

$$
\begin{gathered}
\left.r^{p} \gamma^{j} \operatorname{Pr}\left\{G_{\delta_{0}}\left(X, b_{\left[\gamma^{j}\right.}\right]\right) \geq r \gamma^{j}(\log j)^{1 / 2} a_{\gamma^{j}}^{-1}\right\} \\
\leq \gamma^{j} \operatorname{Pr}\left\{G_{\delta_{0}}\left(X, b_{\left[\gamma^{j}\right]}\right) \geq r_{2} 2^{j}(\log j)^{1 / 2} a_{\left[\gamma^{j}\right]}^{-1}\right\} \\
+r_{1}^{-p} a_{\left[\gamma^{j}\right]}^{p} \gamma^{j(-p+1)}(\log j)^{p / 2} E\left[G_{\delta_{0}}^{p}\left(X, b_{2^{j}}\right) I_{\left.r_{1} \gamma^{j}(\log j)^{-1 / 2} a_{\left[\gamma^{j}\right]}^{-1} \leq G\left(X, b_{\left[\gamma^{j}\right]}\right) \leq r_{2} \gamma^{j}(\log j)^{1 / 2} a_{\left[\gamma^{j}\right]}^{-1}\right]}\right.
\end{gathered}
$$

for $r_{1}(\log j)^{-1} \leq r \leq r_{2}$. So (c.3) implies (c.1). Because $a_{\left[\gamma^{j}\right]}\left[\gamma^{j}\right]^{-1 / 2} G_{\delta_{0}}\left(X, b_{\left[\gamma^{j}\right]}\right) \leq H(X)$ for $j$ large, (c.4) implies that (c.3) is satisfied for any $p>2$. To every particular situation, one of these different conditions can be the most appropriate (see Arcones, 1996b).

Condition (iv) in Theorem 2.4 follows from the following condition:

(iv)' For some $1>\delta_{0}>0$,

$$
\sup _{t \in\left[1-\delta_{0}, 1\right] \times T} a_{n} n^{-1}(2 \log \log n)^{-1 / 2}\left|\sum_{j=1}^{n}\left(g\left(X_{j}, b_{n} t\right)-E\left[g\left(X_{j}, b_{n} t\right)\right]\right)\right| \stackrel{\operatorname{Pr}}{\longrightarrow} 0 .
$$

The notions of VC class and bracketing entropy may be helpful to check last condition. Next theorem follows from Corollary 2.4 in Arcones (1995a).

THEOREM 2.6. With the above notation, suppose that:

(i) The class of functions $\{g(\cdot, u t): t \in T, 0<u \leq 1\}$ is a VC subgraph class of functions.

(ii) $\left\{b_{n}\right\}$ is a non-increasing sequence of numbers from the interval $(0,1]$

(iii) For each $\eta>0$,

$$
n \operatorname{Pr}\left\{\sup _{t \in T} a_{n} n^{-1}(2 \log \log n)^{-1 / 2}\left|g\left(X, b_{n} t\right)\right| \geq \eta\right\} \rightarrow 0 .
$$


(iv) For each $t \in T$,

$$
\lim _{n \rightarrow \infty} a_{n}^{2} n^{-1}(2 \log \log n)^{-1} E\left[\left|g\left(X, b_{n} t\right)\right|^{2} I_{a_{n} n^{-1}(2 \log \log n)^{-1 / 2}\left|g\left(X, b_{n} t\right)\right| \leq 1}\right]=0
$$

(v)

$$
\sup _{n \geq 1} a_{n}^{2} n^{-1}(2 \log \log n)^{-1} E\left[\sup _{t \in T}\left|g\left(X, b_{n} t\right)\right|^{2} I_{a_{n} n^{-1}(2 \log \log n)^{-1 / 2} \sup _{t \in T}\left|g\left(X, b_{n} t\right)\right| \leq 1}\right]<\infty .
$$

(vi) For each $\eta>0$, there exists a finite partition $\pi$ of $T$ such that

$a_{n}^{2} n^{-1}(2 \log \log n)^{-1} \sup _{t \in T} E\left[\left|g\left(X, b_{n} t\right)-g\left(X, b_{n} \pi(t)\right)\right|^{2} I_{a_{n} n^{-1}(2 \log \log n)^{-1 / 2} \sup _{t \in T}\left|g\left(X, b_{n} t\right)\right| \leq 1}\right] \leq \eta$.

(vii)

$$
\lim _{n \rightarrow \infty} \sup _{t \in T} a_{n}(2 \log \log n)^{-1 / 2}\left|E\left[g\left(X, b_{n} t\right) I_{a_{n} n^{-1}(2 \log \log n)^{-1 / 2}\left|g\left(X, b_{n} t\right)\right| \geq 1}\right]\right|=0 .
$$

Then,

$$
\sup _{t \in T} a_{n} n^{-1}(2 \log \log n)^{-1 / 2}\left|\sum_{j=1}^{n}\left(g\left(X_{j}, b_{n} t\right)-E\left[g\left(X_{j}, b_{n} t\right)\right]\right)\right| \stackrel{\operatorname{Pr}}{\longrightarrow} 0 .
$$

Previous theorem improves on Theorem 2.6 in Alexander (1987).

Next theorem follows from Theorem 1.5 in Arcones (1995b).

THEOREM 2.7. With the above notation, suppose that:

(i) $\left\{b_{n}\right\}$ is a non-increasing sequence of numbers from the interval $(0,1]$

(ii) For each $\eta>0$,

$$
n \operatorname{Pr}\left\{\sup _{t \in T} a_{n} n^{-1}(2 \log \log n)^{-1 / 2}\left|g\left(X, b_{n} t\right)\right| \geq \eta\right\} \rightarrow 0 .
$$

(iii) For each $t \in T$,

$$
\lim _{n \rightarrow \infty} a_{n}^{2} n^{-1}(2 \log \log n)^{-1} E\left[\left|g\left(X, b_{n} t\right)\right|^{2} I_{a_{n} n^{-1}(2 \log \log n)^{-1 / 2}\left|g\left(X, b_{n} t\right)\right| \leq 1}\right]=0
$$

(iv)

$$
\lim _{n \rightarrow \infty} \sup _{t \in T} a_{n}(2 \log \log n)^{-1 / 2}\left|E\left[g\left(X, b_{n} t\right) I_{a_{n} n^{-1}(2 \log \log n)^{-1 / 2}\left|g\left(X, b_{n} t\right)\right| \geq 1}\right]\right|=0 .
$$

(v) There are positive integers $q_{0}$ and $n_{0}$; a finite partition $\pi_{q}$ of $T$ for each $q \geq q_{0}$; a function $\gamma_{q}: T \rightarrow[0, \infty)$, for each $q \geq q_{0} ;$ and a function $\Delta_{n, q}\left(\cdot, \pi_{q}(t)\right): S_{n, j} \rightarrow[0, \infty)$, for each $1 \leq j \leq k_{n}$, each $n \geq n_{0}$, each $q \geq q_{0}$ and each $t \in T$; such that

$$
a_{n} n^{-1}(2 \log \log n)^{-1 / 2}\left|g\left(x, b_{n} t\right)-g\left(x, b_{n} \pi_{q}(t)\right)\right| \leq \Delta_{n, q}\left(x, \pi_{q}(t)\right),
$$

for each $x \in S$, each $n \geq n_{0}$, each $q \geq q_{0}$ and each $t \in T$;

$$
\sup _{n \geq n_{0}} n E\left[\left(2^{q} \Delta_{n, q}\left(X, \pi_{q}(t)\right)\right) \wedge\left(2^{2 q} \Delta_{n, q}^{2}\left(X, \pi_{q}(t)\right) I_{\Delta_{n, q}^{2}\left(X, \pi_{q}(t)\right) \leq 1}\right] \leq 1\right.
$$




$$
\lim _{k \rightarrow \infty} \sup _{t \in T} \sum_{q=k}^{\infty} 2^{-q} \gamma_{q}(t)=0
$$

and

$$
\sum_{q=q_{0}}^{\infty} \sum_{t \in T_{q}} e^{-\gamma_{q}^{2}\left(\pi_{q}(t)\right)}<\infty
$$

Then,

$$
\sup _{t \in T} a_{n} n^{-1}(2 \log \log n)^{-1 / 2}\left|\sum_{j=1}^{n}\left(g\left(X_{j}, b_{n} t\right)-E\left[g\left(X_{j}, b_{n} t\right)\right]\right)\right| \stackrel{\operatorname{Pr}}{\longrightarrow} 0 .
$$

When $g(x, \theta)$ is smooth in $\theta$ to check condition (iv) in Theorem 2.4, we can use propositions 1.7 and 1.8 in Arcones (1995b).

Condition (v) in Theorem 2.4 is satisfied if

$$
\lim _{\delta \rightarrow 0} \limsup _{n \rightarrow \infty} \sup _{\substack{s, t \in T \\ \rho(s, t) \leq \delta}} a_{n}^{2} n^{-1} \operatorname{Var}\left(g\left(X, b_{n} s\right)-g\left(X, b_{n} t\right)\right)=0
$$

and

$$
\lim _{\delta \rightarrow 0} \limsup _{n \rightarrow \infty} \sup _{1-\delta \leq u \leq 1} \sup _{t \in T} a_{n}^{2} n^{-1} \operatorname{Var}\left(g\left(X, u b_{n} t\right)-g\left(X, b_{n} t\right)\right)=0
$$

3. The LIL for triangular arrays of empirical processes. Here, we present necessary and sufficient conditions for the L.I.L. of $\left\{U_{n}(t): t \in T\right\}$. The limit set is the unit ball of the reproducing kernel Hilbert space (r.k.h.s.) of certain covariance function. We refer to Aronszajn (1950) for the definition and main properties of r.k.h.s.'s.

Next, we present the main result of this section:

TheOrem 3.1. Let $\left\{X_{j}\right\}_{j=1}^{\infty}$ be a sequence of i.i.d.r.v.'s with values in a measurable space $(S, \mathcal{S})$. Let $T$ be a parameter set. Let $g: S \times T \rightarrow \mathbb{R}$ be a function such that $g(\cdot, t): S \rightarrow \mathbb{R}$ is a measurable function for each $t \in T$. Let $w$ be a positive function defined on $(0,1]$. Let $\left\{b_{n}\right\}$ be a sequence of real numbers from the interval $(0,1]$. Suppose that:

(i) There exists a scalar product defined for each $t \in T$ and each $0 \leq u \leq 1$, so that ut $\in T$.

(ii) For each $s, t \in T$, the following limit exists

$$
R(s, t):=\lim _{u \rightarrow 0+} \frac{\operatorname{Cov}(g(X, u s), g(X, u t))}{w^{2}(u)} .
$$

(iii) $\left\{b_{n}\right\}$ and $\left\{a_{n} n^{-1}(\log \log n)^{-1 / 2}\right\}$ are non-increasing sequences, where $a_{n}=\frac{n^{1 / 2}}{w\left(b_{n}\right)}$.

(iv) $\lim _{\gamma \rightarrow 1+} \lim \sup _{n \rightarrow \infty} \sup _{m: n \leq m \leq \gamma n}\left|a_{n}^{-1} a_{m}-1\right|=0$

and $\lim _{\gamma \rightarrow 1+} \lim \sup _{n \rightarrow \infty} \sup _{m: n \leq m \leq \gamma n}\left|b_{n}^{-1} b_{m}-1\right|=0$.

(v) For each $t \in T, \lim _{u \rightarrow 1-} \rho(u t, t)=0$, where

$$
\rho^{2}(s, t):=R(s, s)+R(t, t)-2 R(s, t) .
$$


(vi)

$$
\lim _{\tau \rightarrow 0+} \limsup _{n \rightarrow \infty} \sup _{t \in T} n^{-1} a_{n}^{2} E\left[\left(g\left(X, b_{n} t\right)-E\left[g\left(X, b_{n} t\right)\right]\right)^{2} I_{G\left(X, b_{n}\right) \geq \tau(\log \log n)^{-1 / 2} a_{n}^{-1} n}\right]=0 .
$$

(vii) There are positive constants $r_{1}$ and $r_{2}$, such that

$$
\sum_{j=2}^{\infty} \sup _{r_{1}(\log j)^{-1} \leq r \leq r_{2}} e^{-r_{1} r^{-1}} 2^{j} \operatorname{Pr}\left\{G\left(X, b_{2^{j}}\right) \geq r 2^{j}(\log j)^{1 / 2} a_{2_{j}}^{-1}\right\}<\infty,
$$

where

$$
G(x, b):=\sup _{t \in T}|g(X, b t)-E[g(X, b t)]| .
$$

Then, the following set of conditions ((a) and (b)) are equivalent:

(a) There is a compact set $C \subset l_{\infty}(T)$ such that, with probability one,

$$
\left\{a_{n}(2 \log \log n)^{-1 / 2}\left(P_{n}-P\right) g\left(\cdot, b_{n} t\right): t \in T\right\}
$$

is relatively compact in $l_{\infty}(T)$ and its limit set is $C$.

(b.1) $(T, \rho)$ is totally bounded.

(b.2) $a_{n}(2 \log \log n)^{-1 / 2} \sup _{t \in T}\left|\left(P_{n}-P\right) g\left(\cdot, b_{n} t\right)\right| \stackrel{\operatorname{Pr}}{\longrightarrow} 0$.

(b.3) For each $\eta>0$, there exists a $\delta>0$ such that

$$
\limsup _{\gamma \rightarrow 1+} \sum_{n=2}^{\infty} \exp \left(-\frac{\eta \log n}{\sup _{\rho(s, t) \leq \delta} s_{\left[\gamma^{n}\right]}^{2}(s, t)}\right)<\infty
$$

where $s_{n}^{2}(s, t)=a_{n}^{2} n^{-1} \operatorname{Var}\left(g\left(X, b_{n} s\right)-g\left(X, b_{n} t\right)\right)$.

Moreover, if either (a) or (b) are satisfied, the limit set $C$ is the unit ball of the r.k.h.s. of the covariance function $R(s, t)$.

Proof. Without loss of generality, we may assume that $E[g(X, t)]=0$ for each $t \in T$.

Assume that the set of conditions (b) holds. We apply Lemma 2.1. In order to get condition (iii) in Lemma 2.1, we apply Theorem 2.4. First, we observe that conditions (i), (ii) and (iv) in Theorem 2.4 are satisfied. Because, $G\left(x, b_{n}\right)$ and $a_{n} n^{-1}(\log \log n)^{-1 / 2}$ are non-increasing, condition (vii) implies that

$$
\sum_{j=2}^{\infty} \sup _{r_{1}(\log j)^{-1} \leq r \leq r_{2}} e^{-r_{1} r^{-1}} \gamma^{j} \operatorname{Pr}\left\{G\left(X, b_{\left[\gamma^{j}\right]}\right) \geq r \gamma^{j}(\log j)^{1 / 2} a_{\left[\gamma^{j}\right]}^{-1}\right\}<\infty,
$$

for any $\gamma>1$. So, condition (iii) in Theorem 2.4 holds.

Next, we will show that the limit covariance is that of a self-similar process. We claim that there exists a constant $\alpha \geq 0$ such that

$$
R(a s, a t)=a^{\alpha} R(s, t) \text { for each } s, t \in T, \text { and each } 1 \geq a \geq 0 .
$$


If $R(t, t)=0$ for each $t \in T,(3.1)$ is trivially true. Assume otherwise, i.e. there exists a $t_{0} \in T$ such that $R\left(t_{0}, t_{0}\right) \neq 0$. First, we will show that $w(u)$ is a function regularly varying at 0 . We have that

$$
\rho^{2}\left(u t_{0}, t_{0}\right)=R\left(u t_{0}, u t_{0}\right)-2 R\left(u t_{0}, t_{0}\right)+R\left(t_{0}, t_{0}\right) \rightarrow 0 \text { as } u \rightarrow 1-.
$$

So, $R\left(u t_{0}, u t_{0}\right) \neq 0$, for each $u$ in a left neighborhood of 1 . We also have that

$$
\lim _{u \rightarrow 0+} \frac{w^{2}(a u)}{w^{2}(u)}=\lim _{u \rightarrow 0+} \frac{w^{2}(a u)}{\operatorname{Var}\left(g\left(X, a u t_{0}\right)\right)} \frac{\operatorname{Var}\left(g\left(X, a u t_{0}\right)\right)}{w^{2}(u)}=\frac{R\left(a t_{0}, a t_{0}\right)}{R\left(t_{0}, t_{0}\right)}
$$

for each $0<a \leq 1$. Hence, by (for example) Theorem 1.4.1 in Bingham, Goldie and Teugels (1987), $w(u)$ is a function regularly varying at 0 and there is a real number $\alpha$ such that

$$
\lim _{u \rightarrow 0+} \frac{w^{2}(a u)}{w^{2}(u)}=a^{\alpha}
$$

for each $a>0$. Because $(T, \rho)$ is totally bounded, $\alpha \geq 0$. Therefore, (3.1) follows.

Next, we prove that

$$
\lim _{u \rightarrow 1-} \sup _{t \in T} \rho(u t, t)=0 .
$$

Given $\delta>0$, take a $\delta$-covering $t_{1}, \ldots, t_{p}$ of $T$, i.e. for each $t \in T$, there is a $1 \leq j \leq p$ such that $\rho\left(t, t_{j}\right) \leq \delta$. We have that

$$
\rho(t, u t) \leq \rho\left(t, t_{j}\right)+\rho\left(t_{j}, u t_{j}\right)+\rho\left(u t_{j}, u t\right) \leq \delta\left(1+u^{\alpha / 2}\right)+\rho\left(t_{j}, u t_{j}\right) .
$$

So, (3.2) follows. From this, we get that condition (v) in Theorem 2.4 is equivalent to (b.3). Therefore, from Theorem 2.4, condition (i) in Lemma 2.1 holds.

Obviously, condition (ii) in this lemma is satisfied.

As to condition (iii) in this lemma. Condition (vi) implies that there exist a sequence $\tau_{n} \searrow 0$ such that

$$
\limsup _{n \rightarrow \infty} \sup _{t \in T} n^{-1} a_{n}^{2} E\left[\left(g\left(X, b_{n} t\right)-E\left[g\left(X, b_{n} t\right)\right]\right)^{2} I_{G\left(X, b_{n}\right) \geq \tau_{n}(\log \log n)^{-1 / 2} a_{n}^{-1} n}\right)=0 .
$$

By the proof in Theorem 2.4, it follows that

$$
\sup _{0 \leq u \leq 1} a_{n}(2 \log \log n)^{-1 / 2}\left|\left(P_{n}-P\right)\left(g\left(\cdot, b_{n} u t\right) I_{G\left(X, b_{n}\right) \geq \tau_{n}(\log \log n)^{-1 / 2} a_{n}^{-1} n}\right)\right| \rightarrow 0 \text { a.s. }
$$

for each $t \in T$. Now that the process is truncated at the right level, the argument in Theorem 3 in Arcones (1994) gives that for each $t_{1}, \ldots, t_{p} \in T,\left\{\left(U_{n}\left(t_{1}\right), \ldots, U_{n}\left(t_{p}\right)\right)\right\}$, is relatively compact in $\mathbb{R}^{p}$ and its limit set is $\left\{\left(x\left(t_{1}\right), \ldots, x\left(t_{p}\right)\right) \in \mathbb{R}^{p}: x \in C\right\}$, where $C$ is the unit ball of the r.k.h.s. of the covariance function $R(s, t)$. So, (b) implies (a).

Assume condition (a). By Lemma 2.2, (b.1), (b.2) and

$$
\lim _{\delta \rightarrow 0} \limsup _{n \rightarrow \infty} \sup _{\substack{s, \in T \\ \rho(s, t) \leq \delta}} a_{n}(\log \log n)^{-1 / 2}\left|\left(P_{n}-P\right)\left(g\left(\cdot, b_{n} s\right)-g\left(\cdot, b_{n} t\right)\right)\right|=0 \quad \text { a.s. }
$$


hold. Condition (vi) and (b.1) imply that there exist a sequence $\tau_{n} \searrow 0$ satisfying (3.4). So, we may assume that

$$
G\left(X, b_{n}\right) \leq \tau_{n}(\log \log n)^{-1 / 2} a_{n}^{-1} n \text { a.s. }
$$

Now, (3.5) and the Kolmogorov zero-one law imply that there exists a constant $c(\delta)$ such that

$$
\limsup _{n \rightarrow \infty} a_{n}(2 \log \log n)^{-1 / 2} \sup _{\rho(s, t) \leq \delta}\left|\left(P_{n}-P\right)\left(g\left(\cdot, b_{n} t\right)-g\left(\cdot, b_{n} s\right)\right)\right|=c(\delta) \text { a.s. }
$$

and $\lim _{\delta \rightarrow 0} c(\delta)=0$. Given $\eta>0$, take $\delta>0$ such that $100 c^{2}(\delta)<\eta$. Let $1<\gamma<8 / 7$ such that

$$
\limsup _{n \rightarrow \infty} \sup _{m: n \leq m \leq[\gamma n]}\left|a_{n}^{-1} a_{m}-1\right| \leq 1 / 2 .
$$

We have that, with probability one, for $n$ large

$$
10^{-1} \eta^{1 / 2}>a_{\left[\gamma^{n}\right]} \gamma^{-n}(\log n)^{-1 / 2} \sup _{\rho(s, t) \leq \delta}\left|\sum_{j=1}^{\left[\gamma^{n}\right]}\left(g\left(X_{j}, b_{\left[\gamma^{n}\right]} t\right)-g\left(X_{j}, b_{\left[\gamma^{n}\right]} s\right)\right)\right|
$$

and

$$
(2 / 5) \eta^{1 / 2}>a_{\left[\gamma^{n}\right]} \gamma^{-n}(\log n)^{-1 / 2} \sup _{\rho(s, t) \leq \delta}\left|\sum_{j=1}^{\left[\gamma^{n-1}\right]}\left(g\left(X_{j}, b_{\left[\gamma^{n}\right]} t\right)-g\left(X_{j}, b_{\left[\gamma^{n}\right]} s\right)\right)\right|
$$

Hence,

$$
a_{\left[\gamma^{n}\right]} \gamma^{-n}(\log n)^{-1 / 2} \sup _{\rho(s, t) \leq \delta}\left|\sum_{j=\left[\gamma^{n-1}\right]+1}^{\left[\gamma^{n}\right]}\left(g_{n}\left(X_{j}, t\right)-g_{n}\left(X_{j}, s\right)\right)\right|<2^{-1} \eta^{1 / 2},
$$

for $n$ large enough, where

$$
g_{n}(x, t):=g\left(x, b_{\left[\gamma^{n}\right]} t\right) I_{G\left(x, b_{\left[\gamma^{n}\right]}\right) \leq \tau_{n}(\log n)^{-1 / 2} a_{\left[\gamma^{n}\right]}^{-1} \gamma^{n}}-E\left[g\left(X, b_{\left[\gamma^{n}\right]} t\right) I_{\left.G\left(X, b_{\left[\gamma^{n}\right]}\right) \leq \tau_{n}(\log n)^{-1 / 2} a_{\left[\gamma^{n}\right]}^{-1} \gamma^{n}\right] .}\right.
$$

By the lemma of Borel-Cantelli

$$
\sum_{n=1}^{\infty} Q_{n}<\infty
$$

where

$$
Q_{n}=\operatorname{Pr}\left\{a_{\left[\gamma^{n}\right]} \gamma^{-n}(\log n)^{-1 / 2} \sup _{\rho(s, t) \leq \delta}\left|\sum_{j=\left[\gamma^{n-1}\right]+1}^{\left[\gamma^{n}\right]}\left(g_{n}\left(X_{j}, t\right)-g_{n}\left(X_{j}, s\right)\right)\right| \geq 2^{-1} \eta^{1 / 2}\right\}
$$

We claim that

$$
\exp \left(-\frac{\eta \log n}{\sup _{\rho(s, t) \leq \delta} \tilde{s}_{\left[\gamma^{n}\right]}^{2}(s, t)}\right) \leq Q_{n}+n^{-2}
$$

for $n$ large, where $\tilde{s}_{n}^{2}(s, t)=a_{n}^{2} n^{-1} \operatorname{Var}\left(g_{n}(X, s)-g_{n}(X, t)\right)$. We apply the Kolmogorov lower bound (see for example Theorem 5.2.2 in Stout, 1974). In the notation in this reference,

$$
\epsilon=2^{-1} \eta^{1 / 2}\left(1-\gamma^{-1}\right)^{-1 / 2}(\log n)^{1 / 2}\left(\tilde{s}_{\left[\gamma^{n}\right]}(s, t)\right)^{-1}
$$


and

$$
c=\tau_{\left[\gamma^{n}\right]}\left(1-\gamma^{-1}\right)^{-1 / 2}(\log n)^{-1 / 2}\left(\tilde{s}_{\left[\gamma^{n}\right]}(s, t)\right)^{-1} .
$$

We need that $\epsilon>\epsilon(1)$ and $\epsilon c \leq \pi(1)$. We have that $\tilde{s}_{n}(s, t) \leq 2 \tau_{n}(\log \log n)^{1 / 2}$. Therefore, $\epsilon>\epsilon(1)$ for $n$ large. If $2^{-1} \tau_{\left[\gamma^{n}\right]}\left(1-\gamma^{-1}\right)^{-1} \eta^{1 / 2}\left(\tilde{s}_{\left[\gamma^{n}\right]}(s, t)\right)^{-2} \leq \pi(1)$, then we may apply the Komogorov lower bound to get that

$$
\exp \left(-\frac{\eta \log n}{\tilde{s}_{\left[\gamma^{n}\right]}^{2}(s, t)}\right) \leq Q_{n}
$$

If $2^{-1} \tau_{\left[\gamma^{n}\right]} \eta^{1 / 2}\left(1-\gamma^{-1}\right)^{-1}\left(\tilde{s}_{\left[\gamma^{n}\right]}(s, t)\right)^{-2}>\pi(1)$, then $\tilde{s}_{\left[\gamma^{n}\right]}^{2}(s, t) \leq \eta / 2$, for $n$ large. So, (3.7) holds.

(b.3) follows from (3.3), (3.6) and (3.7).

Theorem 3.1 is an improvement of Theorem 3 in Arcones (1994). Some applications of last theorem to statistics can be found in Arcones (1994, 1996a, 1996b).

REMARKS 3.2. The part (b) implies (a) is true for any choice of pseudometric $\rho$. For example, in the case that $T$ is a bounded subset of $\mathbb{R}^{d}$, in order to show (a), it suffices to check conditions (i) -(vii) and (b.1)-(b.3) with the Euclidean distance.

Consider the following condition:

(b.4) There exists a finite constant c such that

$$
\sum_{n=3}^{\infty} \operatorname{Pr}\left\{G\left(X, b_{n}\right) \geq c n(\log \log n)^{1 / 2} a_{n}^{-1}\right\}<\infty .
$$

By Lemma 2.2, this condition is a necessary condition for the L.I.L. In some cases, (b.4) implies condition (vii) in Theorem 3.1. Assuming this, and (i)-(vi) in Theorem 3.1, we have that (a) is equivalent to (b.1)-(b.4). For example if there are positive constants $c$ and $q$ such that

$$
k^{-1}\left(k(\log \log k)^{1 / 2} a_{k}^{-1}\right)^{q} \leq c n^{-1}\left(n(\log \log n)^{1 / 2} a_{n}^{-1}\right)^{q}
$$

for each $3 \leq k \leq n$, then condition (b.4) implies condition (vii) in Theorem 3.1. This claim follows from the proof of Lemma 7.8 in Ledoux and Talagrand (1991). Condition (3.8) is satisfied if $n(\log \log n)^{1 / 2} a_{n}^{-1}$ is regularly varying with positive index. In any case, any condition from the Remark 2.5 maybe easier to check in a particular situation. The condition

(c.2)' There exists a sequence $\left\{\tau_{n}\right\}_{n=1}^{\infty}$ of positive numbers converging to zero, such that

$$
a_{n}(\log \log n)^{1 / 2} n^{-1} G\left(X, b_{n}\right) \leq \tau_{n} \text { a.s. }
$$

implies conditions (vi) and (vii) in Theorem 3.1. We also have that condition (c.3) in Remark 2.5 implies conditions (vi) and (vii) in Theorem 3.1.

If we take as an index set $T \subset \bar{T}$, we still can have L.I.L. with limit set being a r.k.h.s.:

THEOREM 3.3. Let $\left\{X_{j}\right\}_{j=1}^{\infty}$ be a sequence of i.i.d.r.v.'s with values in a measurable space $(S, \mathcal{S})$. Let $\bar{T}$ be a parameter set, which has a scalar product defined for each $t \in \bar{T}$ and each 
$0 \leq u \leq 1$. Let $g: S \times \bar{T} \rightarrow \mathbb{R}$ be a function such that $g(\cdot, t): S \rightarrow \mathbb{R}$ is a measurable function for each $t \in \bar{T}$. Let $T \subset \bar{T}$. Let $w$ be a positive function defined on $(0,1]$. Let $\left\{b_{n}\right\}$ be a sequence of real numbers from the interval $(0,1]$. Suppose that:

(i) For each $s, t \in T$, the following limit exists

$$
R(s, t):=\lim _{u \rightarrow 0+} \frac{\operatorname{Cov}(g(X, u s), g(X, u t))}{w^{2}(u)} .
$$

(ii) $\left\{b_{n}\right\}$ and $\left\{a_{n} n^{-1}(\log \log n)^{-1 / 2}\right\}$ are non-increasing sequences, where $a_{n}=\frac{n^{1 / 2}}{w\left(b_{n}\right)}$.

(iii) $\lim _{\gamma \rightarrow 1+} \lim \sup _{n \rightarrow \infty} \sup _{m: n \leq m \leq \gamma n}\left|a_{n}^{-1} a_{m}-1\right|=0$

and $\lim _{\gamma \rightarrow 1+} \lim \sup _{n \rightarrow \infty} \sup _{m: n \leq m \leq \gamma n}\left|b_{n}^{-1} b_{m}-1\right|=0$.

(iv) There are constants $r_{1}, r_{2}>0,1>\delta_{0}>0$, such that

$$
\sum_{j=2}^{\infty} \sup _{r_{1}(\log j)^{-1} \leq r \leq r_{2}} e^{-r_{1} r^{-1}} \gamma^{j} \operatorname{Pr}\left\{G_{\delta_{0}}\left(X, b_{\left[\gamma^{j}\right]}\right) \geq r \gamma^{j}(\log j)^{1 / 2} a_{\left[\gamma^{j}\right]}^{-1}\right\}<\infty,
$$

for each $\gamma>1$.

(v) For each $t \in T$,

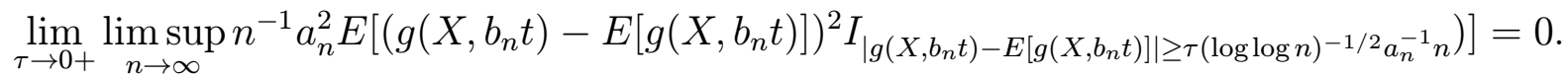

(vi) $(T, \rho)$ is totally bounded.

(vii) For each $\eta>0$,

$$
\limsup _{\delta \rightarrow 0} \lim \operatorname{Pr}\left\{\sup _{1-\delta \leq u \leq 1} \sup _{t \in T}\left|U_{n}(u t)\right| \geq \eta\right\}=0 .
$$

(viii) For each $\eta>0$, there exists a $\delta>0$ such that

$$
\limsup _{\gamma \rightarrow 1+} \sum_{j=2}^{\infty} \exp \left(-\frac{\eta \log j}{\sup _{\substack{s, t \in T \\ \rho(s, t) \leq \delta}} s_{\left[\gamma^{j}\right]}^{2}(s, t)}\right)<\infty,
$$

and

$$
\limsup _{\gamma \rightarrow 1+} \sum_{j=2}^{\infty} \exp \left(-\frac{\eta \log j}{\sup _{t \in T} \sup _{1-\delta \leq u \leq 1} s_{\left[\gamma^{j}\right]}^{2}(u t, t)}\right)<\infty,
$$

where $s_{n}^{2}(s, t)=a_{n}^{2} n^{-1} \operatorname{Var}\left(g\left(X, b_{n} s\right)-g\left(X, b_{n} t\right)\right)$.

Then, with probability one,

$$
\left\{a_{n}(2 \log \log n)^{-1 / 2}\left(P_{n}-P\right) g\left(\cdot, b_{n} t\right): t \in T\right\}
$$

is relatively compact and its limit set is the unit ball of the r.k.h.s. of the covariance function $R(s, t)$.

Proof. We apply Theorem 2.1. The a.s. asymptotic equicontinuity follows from Theorem 2.4. In order to prove the L.I.L for the finite dimensional distributions, we need condition (iv) 
for each $\gamma>1$, because we need blocks $n_{j}=\left[\gamma^{j}\right]$, with $\gamma$ close to one and infinity. We only need condition ( $\mathrm{v}$ ) in the way it is stated, because to prove the L.I.L for the finite dimensional distributions, we only need to truncate for a finite number of $t$ 's.

We also will need the following variation of Theorem 3.3:

THEOREM 3.4. Under the usual notation, suppose that:

(i) For each $s, t \in T$, the following limit exists

$$
R(s, t):=\lim _{u \rightarrow 0+} \frac{\operatorname{Cov}(g(X, u s), g(X, u t))}{w^{2}(u)} .
$$

(ii) $\left\{b_{n}\right\}$ is non-increasing and $\lim _{\gamma \rightarrow 1+} \limsup _{n \rightarrow \infty} \sup _{m: n \leq m \leq \gamma n}\left|b_{n}^{-1} b_{m}-1\right|=0$.

(iii) There are $1<p<\infty$ and $0<a<\infty$ such that

$$
a_{n} n^{-1}(\log \log n)^{p / 2} \rightarrow a,
$$

where $a_{n}=\frac{n^{1 / 2}}{w\left(b_{n}\right)}$.

(iv) There are positive constants $r_{0}>0$ and $\delta_{0}>0$, such that for each $\eta>0$,

$$
\sup \left\{n e^{2 r^{2 /(p+1)}} \operatorname{Pr}\left\{G_{\delta_{0}}\left(X, b_{n}\right) \geq r\right\}: r_{0}(\log \log n)^{(p-1) / 2} \leq r \leq \eta(\log \log n)^{(p+1) / 2}, n \geq 1\right\}<\infty
$$

(v) For each $t \in T$,

$$
\lim _{\tau \rightarrow 0+} \limsup _{n \rightarrow \infty} n^{-1} a_{n}^{2} E\left[\left(g\left(X, b_{n} t\right)-E\left[g\left(X, b_{n} t\right)\right]\right)^{2} I_{G\left(X, b_{n}\right) \geq \tau(\log \log n)^{-1 / 2} a_{n}^{-1} n}\right)=0 .
$$

(vi) $(T, \rho)$ is totally bounded.

(vii) For each $\eta>0$,

$$
\lim _{\delta \rightarrow 0} \limsup _{n \rightarrow \infty} \operatorname{Pr}\left\{\sup _{1-\delta \leq u \leq 1} \sup _{t \in T}\left|U_{n}(u t)\right| \geq \eta\right\}=0 .
$$

(viii) For each $\eta>0$, there exists a $\delta>0$ such that

$$
\lim _{\gamma \rightarrow 1+} \sum_{j=2}^{\infty} \exp \left(-\frac{\eta(\log j)^{p+1}}{\gamma^{j} \sup _{\rho(s, t) \leq \delta} \operatorname{Var}\left(g\left(X, b_{\gamma^{j}} s\right)-g\left(X, b_{\gamma^{j}} t\right)\right)}\right)
$$

and

$$
\lim _{\gamma \rightarrow 1+} \sum_{j=2}^{\infty} \exp \left(-\frac{\eta(\log j)^{p+1}}{\gamma^{j} \sup _{1-\delta \leq u \leq 1} \sup _{t \in T} \operatorname{Var}\left(g\left(X, u b_{\gamma^{j}} t\right)-g\left(X, b_{\gamma^{j}} t\right)\right)}\right) .
$$

Then, with probability one,

$$
\left\{a_{n}(2 \log \log n)^{-1 / 2}\left(P_{n}-P\right) g\left(\cdot, b_{n} t\right): t \in T\right\}
$$

is relatively compact and its limit set is the unit ball of the r.k.h.s. of the covariance function $R(s, t)$. 
Proof. Without loss of generality, we may assume that $E[g(X, t)]=0$ for each $t \in T$. The proof is exactly the same as that of Theorem 3.3. The difference is in the bound in the term $\operatorname{Pr}\left\{\sum_{i=1}^{k} Y_{n_{j}, i}^{*} \geq s\right\}$ in the Talagrand isoperimetric inequality. Take $1<r<(p+1) / 2$. Let $\zeta=\sum_{i=1}^{\infty} i^{-r}$. We want to show that for each $\eta>0$, there exists a $\delta>0$ and a $\gamma>1$, such that

$$
\begin{gathered}
\sum_{j=1}^{\infty} \operatorname{Pr}\left\{\sup _{\rho(s, t) \leq \delta}\left|\sum_{i=1}^{n_{j}} \epsilon_{i}\left(g\left(X_{i}, b_{n_{j}} s\right)-g\left(X_{i}, b_{n_{j}} t\right)\right)\right|\right. \\
\left.\geq\left(16 K_{0}^{1 / 2} \eta+8 \zeta \eta+8 r\left(\log \eta^{-1}\right)^{-1}\right)(\log j)^{(p+1) / 2}\right\}<\infty,
\end{gathered}
$$

where $n_{j}=\left[\gamma^{j}\right]$. To prove this we apply Theorem 2.3 with

$$
\begin{gathered}
q=K_{0} \eta^{-1}, k=\left[2(\log j)\left(\log \eta^{-1}\right)^{-1}\right] \\
t=16 K_{0}^{1 / 2} \eta(\log j)^{(p+1) / 2} \text { and } s=\left(2 \zeta \eta+4 r\left(\log \eta^{-1}\right)^{-1}\right)(\log j)^{(p+1) / 2}
\end{gathered}
$$

Let $l_{j}=r^{-2 /(p+1)}(\log j)^{2 /(p+1)}$. We have that if $Y_{n_{j}, i}^{*} \leq 2 i^{-r} \eta(\log j)^{(p+1) / 2}$ for $1 \leq i \leq l_{j}$ and $Y_{n_{j}, l_{j}}^{*} \leq 2 r(\log j)^{(p-1) / 2}$, then

$$
\sum_{i=1}^{k} Y_{n_{j}, i}^{*} \leq s
$$

Since

$$
\operatorname{Pr}\left\{Y_{n_{j}, i}^{*} \geq 2 t\right\} \leq\left(\operatorname{Pr}\left\{Y_{n_{j}, 1}^{*} \geq 2 t\right\}\right)^{i} \leq\left(n \operatorname{Pr}\left\{G\left(X, b_{n}\right) \geq t\right\}\right)^{i},
$$

(see the proof of Theorem 6.20 in Ledoux and Talagrand (1991)) we have that

$$
\begin{aligned}
\operatorname{Pr}\left\{\sum_{i=1}^{k} Y_{n_{j}, i}^{*} \geq s\right\} \leq & \sum_{i=1}^{l_{j}} \operatorname{Pr}\left\{Y_{n_{j}, i}^{*} \geq 2 i^{-r} \eta(\log j)^{(p+1) / 2}\right\}+\operatorname{Pr}\left\{Y_{n_{j}, l_{j}}^{*} \geq 2 r(\log j)^{(p-1) / 2}\right\} \\
& \leq(\theta-1)^{-1} \theta^{l_{j}-2 \log j}+\theta e^{-2 \log j} \leq O\left(j^{-3 / 2}\right)
\end{aligned}
$$

where

$$
\theta:=2+\sup \left\{n e^{2 r^{2 /(p+1)}} \operatorname{Pr}\left\{G\left(X, b_{n}\right) \geq r\right\}: r_{0}(\log \log n)^{(p-1) / 2} \leq r \leq \eta(\log \log n)^{(p+1) / 2}\right\} .
$$

The rest of the estimations are as in previous proofs and are omitted.

4. The L.I.L. for density estimators. First, we consider the L.I.L. for the stochastic processes in (1.2).

TheOREM 4.1. Let $\left\{X_{j}\right\}_{j=1}^{\infty}$ be a sequence of i.i.d.r.v.'s with values in $\mathbb{R}^{d}$, let $\left\{K_{t}(\cdot): t \in T\right\}$ be a class of measurable function on $\mathbb{R}^{d}$, let $M<\infty$, let $\lambda$ be the Lebesgue measure on $\mathbb{R}^{d}$, let $x_{0} \in \mathbb{R}^{d}$ and let $\left\{b_{n}\right\}$ be a sequence of positive numbers. Suppose that:

(i) $b_{n} \searrow 0$ and $\frac{\log \log n}{b_{n}^{d} n} \searrow 0$.

(ii) For each $t \in T, K_{t}(x)=0$ if $|x|>M$, and $\int_{\mathbb{R}^{d}} K_{t}^{2}(x) d x<\infty$. 
(iii) There are $r_{1}, r_{2}>0$ and $1>\delta_{0}>0$, such that

$$
\sum_{j=2}^{\infty} \sup _{r_{1}(\log j)^{-1} \leq r \leq r_{2}} e^{-r_{1} r^{-1}} 2^{j} b_{2^{j}}^{d} \lambda\left\{x \in \mathbb{R}^{d}: G_{\delta_{0}}(x) \geq r 2^{j / 2}(\log j)^{1 / 2} b_{2^{j}}^{d / 2}\right\}<\infty
$$

where

$$
G_{\delta_{0}}(x):=\sup _{1-\delta_{0} \leq u \leq 1} \sup _{t \in T}\left|K_{t}\left(u^{-1} x\right)\right| .
$$

(iv) $X$ has a density $f$ in a neighborhood of $x_{0}$, which is continuous at $x_{0}$.

(v) $(T, \rho)$ is totally bounded, where $\rho^{2}(s, t):=\int_{\mathbb{R}^{d}}\left(K_{s}(x)-K_{t}(x)\right)^{2} d x$.

(vi) For each $\eta>0$,

$$
\lim _{\delta \rightarrow 0} \limsup _{n \rightarrow \infty} \operatorname{Pr}\left\{\sup _{1-\delta \leq u \leq 1} \sup _{t \in T}\left|V_{n}(u t)\right| \geq \eta\right\}=0
$$

where

$$
V_{n}(t):=\left(n b_{n}^{d} 2 \log \log n\right)^{-1 / 2} \sum_{j=1}^{n}\left(K_{t}\left(b_{n}^{-1}\left(x_{0}-X_{j}\right)\right)-E\left[K_{t}\left(b_{n}^{-1}\left(x_{0}-X_{j}\right)\right)\right]\right) .
$$

Then, with probability one, $\left\{V_{n}(t): t \in T\right\}$ is relatively compact in $l_{\infty}(T)$ and its limit set is

$$
C:=\left\{\left(\left(f\left(x_{0}\right)\right)^{1 / 2} \int_{\mathbb{R}^{d}} K_{t}(x) \alpha(x) d x\right)_{t \in T}: \int_{\mathbb{R}^{d}} \alpha^{2}(x) d x \leq 1\right\} .
$$

Proof. We apply Theorem 3.3. Let $\bar{T}=\{u t: 0 \leq u \leq 1, t \in T\}$, let $w(u)=u^{d / 2}$, let $a_{n}=b_{n}^{-d / 2} n^{1 / 2}$ and let $g(x, u t)=K_{t}\left(u^{-1}\left(x_{0}-x\right)\right)$. By a change of variables

$$
\begin{gathered}
u^{-d} E[g(X, u s) g(X, u t)]=u^{-d} \int_{\left|x_{0}-x\right| \leq u M} K_{s}\left(u^{-1}\left(x_{0}-x\right)\right) K_{t}\left(u^{-1}\left(x_{0}-x\right)\right) f(x) d x \\
=\int_{|y| \leq M} K_{s}(y) K_{t}(y) f\left(x_{0}-u y\right) d y \rightarrow \int_{|y| \leq M} K_{s}(y) K_{t}(y) f\left(x_{0}\right) d y,
\end{gathered}
$$

as $u \rightarrow 0+$. We also have that

$$
u^{-d} E[g(X, u s)] E[g(X, u t)]=u^{d} \int_{|y| \leq M} K_{s}(y) f\left(x_{0}-u y\right) d y \int_{|y| \leq M} K_{t}(y) f\left(x_{0}-u y\right) d y \rightarrow 0 .
$$

Therefore,

$$
\lim _{u \rightarrow 0+} u^{-d} \operatorname{Cov}(g(X, u s), g(X, u t))=f\left(x_{0}\right) \int_{\mathbb{R}^{d}} K_{s}(x) K_{t}(x) d x,
$$

i.e. condition (i) in Theorem 3.3 holds. It is easy to see that conditions (ii)-(vii) in this theorem hold. By the argument in (4.1), there exists and integer $n_{0}$ and a finite constant $c$, such that

$$
b_{n}^{-d} E\left[\left(K_{s}\left(b_{n}^{-1}\left(x_{0}-X\right)\right)-K_{t}\left(b_{n}^{-1}\left(x_{0}-X\right)\right)\right)^{2}\right] \leq c \int_{x \in R^{d}}\left(K_{t}(x)-K_{s}(x)\right)^{2} d x
$$

for each $s, t \in T$ and each $n \geq n_{0}$. So,

$$
\lim _{\delta \rightarrow 0} \limsup _{n \rightarrow \infty} \sup _{\rho(s, t) \leq \delta} b_{n}^{-d} E\left[\left(K_{s}\left(b_{n}^{-1}\left(x_{0}-X\right)\right)-K_{t}\left(b_{n}^{-1}\left(x_{0}-X\right)\right)\right)^{2}\right]=0 .
$$


We claim that

$$
\lim _{u \rightarrow 1-} \int_{\mathbb{R}^{d}}\left(K_{t}\left(u^{-1} x\right)-K_{t}(x)\right)^{2} d x=0
$$

for each each $t \in T$. By the dominated convergence theorem, (4.2) holds for each bounded, continuous function $K_{t}$. By the Lusin theorem, (4.2) holds for each function $K_{t}$ with $\int_{\mathbb{R}^{d}} K_{t}^{2}(x) d x<\infty$. This and the totally boundedness of $(T, \rho)$, imply that

$$
\lim _{\delta \rightarrow 0} \limsup _{n \rightarrow \infty} \sup _{1-\delta \leq u \leq 1} \sup _{t \in T} b_{n}^{-d} E\left[\left(K_{t}\left(u^{-1} b_{n}^{-1}\left(x_{0}-X\right)\right)-K_{t}\left(b_{n}^{-1}\left(x_{0}-X\right)\right)^{2}\right]=0 .\right.
$$

Therefore, the result follows.

REMARKS 4.2. If $\left\{K_{t}\left(u^{-1} x\right): 0 \leq u \leq 1, t \in T\right\}=\left\{K_{t}(x): t \in T\right\}$, and conditions (i)-(iv) in the Theorem 4.1 are satisfied, then Theorem 3.1 gives that $\left\{V_{n}(t): t \in T\right\}$ satisfies the compact L.I.L. if and only if $(T, \rho)$ is totally bounded and $\sup _{t \in T}\left|V_{n}(t)\right| \stackrel{\mathrm{Pr}}{\longrightarrow} 0$. Condition (iii) in Theorem 4.1 is a moment condition.

Under $\left\{K_{t}\left(u^{-1} x\right): 0 \leq u \leq 1, t \in T\right\}=\left\{K_{t}(x): t \in T\right\}$, by Lemma 2.2, a necessary condition for the L.I.L. is that there exists a finite constant $c$ such that

$$
\sum_{n=3}^{\infty} \operatorname{Pr}\left\{G\left(X, b_{n}\right) \geq c(\log \log n)^{1 / 2} b_{n}^{d / 2} n^{1 / 2}\right\}<\infty .
$$

This implies that there exists a constant $c$ (possibly different) such that

$$
\sum_{n=3}^{\infty} b_{n}^{d} \lambda\left\{x \in \mathbb{R}^{d}: G(x) \geq c(\log \log n)^{1 / 2} b_{n}^{d / 2} n^{1 / 2}\right\}<\infty,
$$

where $G(x)=\sup _{0 \leq u \leq 1} \sup _{t \in T}\left|K_{t}\left(u^{-1} x\right)\right|$. It is easy to see that if $\left\{b_{n}^{d}\right\}$ is regularly varying with index $p>-1$, then (4.3) is equivalent to

$$
\int_{\mathbb{R}^{d}} G_{\delta_{0}}^{2}(x)\left(\log \log \left(G_{\delta_{0}}(x)+9\right)\right)^{-1} d x<\infty .
$$

We also have that if $\left\{b_{n}^{d}\right\}$ is regularly varying with index $p>-1$, then (4.4) is equivalent to condition (iii) in Theorem 4.3. To see this, we will need the following proposition:

Proposition 4.3. With the notation in Theorem 4.1, suppose that:

(i) $b_{n} \searrow 0$.

(ii) There are constants $\tau, p>0$ such that

$$
a_{n} / a_{k} \geq \tau(n / k)^{p},
$$

for each $9 \leq k \leq n$, where $a_{n}=n b_{n}^{d} \log \log n$.

(iii)

$$
\sum_{n=5}^{\infty} 2^{n / 2} b_{2^{n}}^{d / 2} \lambda\left\{x \in \mathbb{R}^{d}: G_{\delta_{0}}(x) \geq a_{2^{n}}^{1 / 2}\right\}<\infty .
$$


Then,

$$
\sum_{n=2}^{\infty} \sup _{r_{1}(\log n)^{-1} \leq r \leq r_{2}} e^{-r_{1} r^{-1}} 2^{n} b_{2^{n}}^{d} \lambda\left\{x \in \mathbb{R}^{d}: G_{\delta_{0}}(x) \geq r a_{2^{n}}^{1 / 2}\right\}<\infty
$$

for each $r_{1}, r_{2}>0$.

Proof. Let $\gamma_{n}=2^{n / 2} b_{2^{n}}^{d / 2} \lambda\left\{x \in \mathbb{R}^{d}: G_{\delta_{0}}(x) \geq a_{2^{n}}^{1 / 2}\right\}$. Let $\beta_{n}=\sum_{j=0}^{\infty} 2^{-j} \gamma_{n+j}$. It is easy to see that $\gamma_{n} \leq \beta_{n}, \beta_{n} \leq 2 \beta_{n+1}$ and $\sum_{n=1}^{\infty} \beta_{n}<\infty$. Let $r_{1}(\log j)^{-1} \leq r \leq r_{2}$, then there exists a integer $k$ such that

$$
2^{-k p / 2} \leq \tau^{1 / 2} r \leq 2^{(-k+1) p / 2}
$$

Since $2^{-n p / 2}<\tau^{1 / 2} r_{1}(\log n)^{-1}, 1 \leq k \leq n$. We have that

$$
r a_{2^{n}}^{1 / 2} \geq \tau^{-1 / 2} 2^{-k p / 2} a_{2^{n}}^{1 / 2} \geq a_{2^{n-k}}^{1 / 2} .
$$

Thus,

$$
\begin{aligned}
& e^{-r_{1} r^{-1}} 2^{n} b_{2^{n}}^{d} \lambda\left\{x \in \mathbb{R}^{d}: G_{\delta_{0}}(x) \geq r a_{2^{n}}^{1 / 2}\right\} \geq e^{-r_{1} r^{-1}} 2^{n} b_{2^{n}}^{d} \lambda\left\{x \in \mathbb{R}^{d}: G_{\delta_{0}}(x) \geq a_{2^{n-k}}^{d / 2}\right\} \\
& \leq e^{-r_{1} r^{-1}} 2^{k} b_{2^{n}}^{d} \beta_{n-k} b_{2^{k}}^{-d} \leq e^{-r_{1} c^{1 / 2} 2^{(k-1) p / 2}+2 k} \beta_{n} \leq c \beta_{n},
\end{aligned}
$$

for a universal constant $c$. So, the claim follows.

It follows from previous proposition that if $\left\{b_{n}\right\}$ is regularly varying with order $p$ for some $0>p>-1$ (and conditions (i) and (ii) in Proposition 4.3 are satisfied), then (4.4) is equivalent to condition (iii) in Theorem 4.1 .

If

$$
b_{n}^{d} n(\log \log n)^{-p} \rightarrow a,
$$

for some finite positive constant $a$ and some $p>0$, then condition (4.3) is equivalent to

$$
\int_{\mathbb{R}^{d}} \exp \left(c G^{2 /(p+1)}(x)\right) d x<\infty
$$

for some $c<\infty$. Next theorem follows from Theorem 3.4.

TheOrem 4.4. Let $\left\{X_{j}\right\}_{j=1}^{\infty}$ be a sequence of i.i.d.r.v.'s with values in $\mathbb{R}^{d}$, let $\left\{K_{t}(\cdot): t \in T\right.$ be a class of measurable function on $\mathbb{R}^{d}$, let $M<\infty$, let $\lambda$ be the Lebesgue measure on $\mathbb{R}^{d}$, let $x_{0} \in \mathbb{R}^{d}$ and let $\left\{b_{n}\right\}$ be a sequence of positive numbers. Suppose that:

(i) For each $t \in T, K_{t}(x)=0$ if $|x|>M$, and $\int_{\mathbb{R}^{d}} K_{t}^{2}(x) d x<\infty$.

(ii) There are constants $0<a<\infty$ and $1<p<\infty$ such that

$$
(\log \log n)^{-p} n b_{n}^{d} \rightarrow a
$$

(iii) There exists $1>\delta_{0}>0$ such that, for each $0<u<\infty$,

$$
\int_{|x| \leq M} \exp \left(u G_{\delta_{0}}^{2 /(p+1)}(x)\right) d x<\infty .
$$


(iv) $X$ has a density $f$ in a neighborhood of $x_{0}$, which is continuous at $x_{0}$.

(v) $(T, \rho)$ is totally bounded, where $\rho^{2}(s, t):=\int_{\mathbb{R}^{d}}\left(K_{s}(x)-K_{t}(x)\right)^{2} d x$.

(vi) For each $\eta>0$,

$$
\lim _{\delta \rightarrow 0} \limsup _{n \rightarrow \infty} \operatorname{Pr}\left\{\sup _{1-\delta \leq u \leq 1} \sup _{t \in T}\left|V_{n}(u t)\right| \geq \eta\right\}=0 .
$$

Then, with probability one, $\left\{V_{n}(t): t \in T\right\}$ is relatively compact in $l_{\infty}(T)$ and its limit set is

$$
\left\{\left(\left(f\left(x_{0}\right)\right)^{1 / 2} \int_{\mathbb{R}^{d}} K_{t}(x) \alpha(x) d x\right)_{t \in T}: \int_{\mathbb{R}^{d}} \alpha^{2}(x) d x \leq 1\right\} .
$$

Theorems 2.6 and 2.7 give sufficient conditions for condition (vi) in Theorem 4.1. For example, $\left\{I_{|x| \leq u}: 0 \leq u \leq 1\right\}$ be a VC subgraph class of functions. Let $\left\{b_{n}\right\}$ be a sequence satisfying condition (i) in Theorem 4.1. Then, by theorems 4.1 and 2.6, with probability one,

$$
\left\{\left(n b_{n}^{d} 2 \log \log n\right)^{-1 / 2} \sum_{j=1}^{n}\left(I_{\left|x_{0}-X_{j}\right| \leq u b_{n}}-\operatorname{Pr}\left\{\left|x_{0}-X_{j}\right| \leq u b_{n}\right\}\right): 0 \leq u \leq 1\right\}
$$

is relatively compact and its limit set is

$$
C:=\left\{\left(\left(f\left(x_{0}\right)\right)^{1 / 2} \int_{|x| \leq u} \alpha(x) d x\right)_{0 \leq u \leq 1}: \int_{\mathbb{R}^{d}} \alpha^{2}(x) d x \leq 1\right\} .
$$

When $T$ has only one element, the conditions given by these theorems simplify. Next, we state what we obtain for a unique kernel.

THEOREM 4.5. Let $\left\{X_{j}\right\}_{j=1}^{\infty}$ be a sequence of i.i.d.r.v.'s with values in $\mathbb{R}^{d}$, let $K(\cdot)$ be a measurable function on $\mathbb{R}^{d}$, let $M<\infty$, let $\lambda$ be the Lebesgue measure on $\mathbb{R}^{d}$, let $x_{0} \in \mathbb{R}^{d}$ and let $\left\{b_{n}\right\}$ be a sequence of positive numbers. Suppose that:

(i) $b_{n} \searrow 0$ and $\frac{\log \log n}{b_{n}^{d} n} \searrow 0$.

(ii) $K(x)=0$ if $|x|>M$, and $\int_{\mathbb{R}^{d}} K^{2}(x) d x<\infty$.

(iii) There are $r_{1}, r_{2}>0$ and $1>\delta_{0}>0$, such that

$$
\sum_{j=2}^{\infty} \sup _{r_{1}(\log j)^{-1} \leq r \leq r_{2}} e^{-r_{1} r^{-1}} 2^{j} b_{2^{j}}^{d} \lambda\left\{x \in \mathbb{R}^{d}: \sup _{1-\delta_{0} \leq u \leq 1}\left|K\left(u^{-1} x\right)\right| \geq r 2^{j / 2}(\log j)^{1 / 2} b_{2^{j}}^{d / 2}\right\}<\infty .
$$

(iv) $X$ has a density $f$ in a neighborhood of $x_{0}$, which is continuous at $x_{0}$.

(v) The class of functions $\left\{K\left(u^{-1} \cdot\right): 0 \leq u \leq 1\right\}$ is a VC subgraph class of functions.

(vi)

$$
\begin{gathered}
\lim _{\delta \rightarrow 0+} \limsup _{n \rightarrow \infty}(2 \log \log n)^{-1} \int_{x \in[-M, M]^{d}} \sup _{1-\delta \leq u \leq 1}\left(K\left(u^{-1} x\right)-K(x)\right)^{2} \\
\times I_{\sup _{1-\delta_{0} \leq u \leq 1}\left|K\left(u^{-1} x\right)\right| \leq n^{1 / 2} b_{n}^{d / 2}(2 \log \log n)^{1 / 2}} d x=0 .
\end{gathered}
$$

Then, with probability one,

$$
\left(n b_{n}^{d} 2 \log \log n\right)^{-1 / 2} \sum_{j=1}^{n}\left(K\left(b_{n}^{-1}\left(x_{0}-X_{j}\right)\right)-E\left[K\left(b_{n}^{-1}\left(x_{0}-X_{j}\right)\right)\right]\right)
$$


is relatively compact and its limit set is

$$
C:=\left\{\left(f\left(x_{0}\right)\right)^{1 / 2} \int_{\mathbb{R}^{d}} K(x) \alpha(x) d x: \int_{\mathbb{R}^{d}} \alpha^{2}(x) d x \leq 1\right\} .
$$

Observe that if $\log \log \left(b_{n}^{d} n\right)=O(\log \log n)$, then condition (vi) in Theorem 4.5 is satisfied if

$$
\lim _{\delta \rightarrow 0+} \int_{x \in[-M, M]^{d}}\left(\log \log \left(9+\sup _{1-\delta \leq u \leq 1}\left|K\left(u^{-1} x\right)-K(x)\right|\right)\right)^{-1} \sup _{1-\delta \leq u \leq 1}\left(K\left(u^{-1} x\right)-K(x)\right)^{2} d x=0 .
$$

If $b_{n}^{d} n(\log \log n)^{-p} \rightarrow a$, for some finite positive constant $a$ and some $p>1$, then condition (vi) in Theorem 4.5 is satisfied if

$$
\lim _{\delta \rightarrow 0+} \sup _{t>0} t^{2 p /(p+1)} \lambda\left\{x \in[-M, M]^{d}: \sup _{1-\delta \leq u \leq 1}\left|K\left(u^{-1} x\right)-K(x)\right| \geq t\right\}=0 .
$$

In general, condition (vi) in Theorem 4.5 is between the second moment and the first moment.

In the bracketing case, we have the following:

THEOREM 4.6. Let $\left\{X_{j}\right\}_{j=1}^{\infty}$ be a sequence of i.i.d.r.v.'s with values in $\mathbb{R}^{d}$, let $K(\cdot)$ be a measurable function on $\mathbb{R}^{d}$, let $M<\infty$, let $\lambda$ be the Lebesgue measure on $\mathbb{R}^{d}$, let $x_{0} \in \mathbb{R}^{d}$ and let $\left\{b_{n}\right\}$ be a sequence of positive numbers. Suppose that:

(i) $b_{n} \searrow 0$ and $\frac{\log \log n}{b_{n}^{d} n} \searrow 0$.

(ii) $K(x)=0$ if $|x|>M$, and $\int_{\mathbb{R}^{d}} K^{2}(x) d x<\infty$.

(iii) There are $r_{1}, r_{2}>0$ and $1>\delta_{0}>0$, such that

$$
\sum_{j=2}^{\infty} \sup _{r_{1}(\log j)^{-1} \leq r \leq r_{2}} e^{-r_{1} r^{-1}} 2^{j} b_{2^{j}}^{d} \lambda\left\{x \in \mathbb{R}^{d}: \sup _{1-\delta_{0} \leq u \leq 1}\left|K\left(u^{-1} x\right)\right| \geq r 2^{j / 2}(\log j)^{1 / 2} b_{2^{j}}^{d / 2}\right\}<\infty .
$$

(iv) $X$ has a density $f$ in a neighborhood of $x_{0}$, which is continuous at $x_{0}$.

(v) There are $c, v>0$ such that

$$
\begin{gathered}
n b_{n}^{d} \int_{x \in[-M, M]^{d}}\left(\left(n b_{n}^{d} \log \log n\right)^{-1 / 2} \sup _{1-\delta \leq u \leq 1}\left|K\left(u^{-1} x\right)-K(x)\right|\right) \\
\wedge\left(\left(n b_{n}^{d} \log \log n\right)^{-1} \sup _{1-\delta \leq u \leq 1}\left(K\left(u^{-1} x\right)-K(x)\right)^{2}\right) d x \leq c \delta^{v},
\end{gathered}
$$

for each $\delta>0$ small enough and each $n$ large enough.

Then, with probability one,

$$
\left(n b_{n}^{d} 2 \log \log n\right)^{-1 / 2} \sum_{j=1}^{n}\left(K\left(b_{n}^{-1}\left(x_{0}-X_{j}\right)\right)-E\left[K\left(b_{n}^{-1}\left(x_{0}-X_{j}\right)\right)\right]\right)
$$

is relatively compact and its limit set is

$$
C:=\left\{\left(f\left(x_{0}\right)\right)^{1 / 2} \int_{\mathbb{R}^{d}} K(x) \alpha(x) d x: \int_{\mathbb{R}^{d}} \alpha^{2}(x) d x \leq 1\right\}
$$


As before, we have that condition (v) in Theorem 4.6 is a tail condition, between the second moment and the first moment.

Next, we present a law of the iterated logarithm of a class of density kernel estimators:

ThEOREM 4.7. Under the conditions in Theorem 4.1, suppose also that (ii) and (iv) are reinforced to:

(ii)' For each $t \in T, K_{t}(x)=0$ if $|x|>M, \int_{\mathbb{R}^{d}} K_{t}(x) d x=1, \int_{\mathbb{R}^{d}} x K_{t}(x) d x=0$ and $\int_{\mathbb{R}^{d}} K_{t}^{2}(x) d x<\infty$.

(iv)' $X$ has a density $f$ in a neighborhood of $x_{0}$, which is second differentiable at $x_{0}$.

Then,

(a) If $n b_{n}^{d+4}(\log \log n)^{-1} \rightarrow 0$, then, with probability one,

$$
\left\{n^{1 / 2} b_{n}^{d / 2}(2 \log \log n)^{-1 / 2}\left(n^{-1} b_{n}^{-d} \sum_{j=1}^{n} K_{t}\left(b_{n}^{-1}\left(x_{0}-X_{j}\right)\right)-f\left(x_{0}\right)\right): t \in T\right\},
$$

is relatively compact and its limit set is

$$
\left\{\left(\left(f\left(x_{0}\right)\right)^{1 / 2} \int_{\mathbb{R}^{d}} K_{t}(x) \alpha(x) d x\right)_{t \in T}: \int_{\mathbb{R}^{d}} \alpha^{2}(x) d x \leq 1\right\} .
$$

(b) If $n b_{n}^{d+4}(\log \log n)^{-1} \rightarrow a$, for some constant $a, 0<a<\infty$, then, with probability one,

$$
\left\{n^{1 / 2} b_{n}^{d / 2}(2 \log \log n)^{-1 / 2}\left(n^{-1} b_{n}^{-d} \sum_{j=1}^{n} K_{t}\left(b_{n}^{-1}\left(x_{0}-X_{j}\right)\right)-f\left(x_{0}\right)\right): t \in T\right\},
$$

is relatively compact and its limit set is

$$
\left\{\left(\int_{\mathbb{R}^{d}} K_{t}(x)\left(\left(f\left(x_{0}\right)\right)^{1 / 2} \alpha(x)+a^{1 / 2} 2^{-1 / 2} x^{\prime} f^{\prime \prime}\left(x_{0}\right) x\right) d x\right)_{t \in T}: \int_{\mathbb{R}^{d}} \alpha^{2}(x) d x \leq 1\right\} .
$$

(c) If $n b_{n}^{d+4}(\log \log n)^{-1} \rightarrow \infty$, then

$$
\lim _{n \rightarrow 0} \sup _{t \in T}\left|n^{-1} b_{n}^{-d-1} \sum_{j=1}^{n} K_{t}\left(b_{n}^{-1}\left(x_{0}-X_{j}\right)\right)-b_{n}^{-1} f\left(x_{0}\right)-\int_{\mathbb{R}^{d}} f(x) 2^{-1 / 2} x^{\prime} f^{\prime \prime}\left(x_{0}\right) x d x\right|=0 \text { a.s. }
$$

Proof. We have that

$$
\begin{gathered}
n^{-1} b_{n}^{-d} \sum_{j=1}^{n} K_{t}\left(b_{n}^{-1}\left(\theta_{0}-X_{j}\right)\right)-f\left(x_{0}\right) \\
=n^{-1} b_{n}^{-d} \sum_{j=1}^{n}\left(K_{t}\left(b_{n}^{-1}\left(x_{0}-X_{j}\right)\right)-E\left[K_{t}\left(b_{n}^{-1}\left(x_{0}-X_{j}\right)\right)\right]\right) \\
+\int_{|x| \leq M} K_{t}(x)\left(f\left(x_{0}-b_{n} x\right)-f\left(x_{0}\right)-\left(f^{\prime}\left(x_{0}\right)\right) x\right) d x .
\end{gathered}
$$

This and Theorem 4.1 imply the claim. 
Observe that the higher order of convergence is attained for

$$
b_{n} \simeq(\log \log n)^{1 /(d+4)} n^{-1 /(d+4)} .
$$

This result is related with a result on the optimal window in the convergence in distribution of density estimators in Parzen (1962).

5. The L.I.L. for triangular arrays of empirical processes in the Poisson domain of attraction. We study the law of the iterated logarithm for a triangular arrays of empirical processes in the Poisson domain, i.e. the limit set is the limit set appearing in the L.I.L. of certain Poisson process. First, we consider the case of one function. This problem has been considered by Deheuvels and Mason $(1990,1991,1995)$ in the case that $d=1$ for a sequence of i.i.d.r.v.'s with uniform distribution on $[0,1]$. We use a large deviations approach as in these references. A reference in large deviations is Dembo and Zeitouni (1993). To avoid repetitions, here is the framework that we will use:

SET-UP. Let $\left\{X_{j}\right\}_{j=1}^{\infty}$ be a sequence of i.i.d.r.v.'s with values in $\mathbb{R}^{d}$, let $X$ be a copy of $X_{1}$, let $M<\infty$, let $x_{0} \in \mathbb{R}^{d}$, let $\left\{b_{n}\right\}$ be a sequence of positive numbers, let $K$ be a measurable function on $\mathbb{R}^{d}$ and let $\left\{K_{t}: t \in T\right\}$ be a class of functions on $\mathbb{R}^{d}$. Assume that:

(c.1) There exists a positive number a, such that $n^{-1} b_{n}^{-d} \log \log n \rightarrow a$.

(c.2) $X$ has a density $f$ in a neighborhood of $x_{0}$, which is continuous and positive at $x_{0}$.

We study the logarithm of the iterated logarithm of processes of the form $\left\{V_{n}\left(K_{t}\right): t \in T\right\}$, where

$$
V_{n}\left(K_{t}\right):=a(\log \log n)^{-1} \sum_{j=1}^{n} K_{t}\left(b_{n}^{-1}\left(x_{0}-X_{j}\right)\right)
$$

and $\left\{K_{t}: t \in T\right\}$ is a class of functions on $\mathbb{R}^{d}$.

First, we present a lemma on the large deviations of $V_{n}(K)$.

Lemma 5.1. With the notation in the Set-up, assume conditions (c.1), (c.2),

(c.3) $K(x)=0$, if $|x|>M$.

(c.4) There exists a positive constant $r_{0}$ such that $\int_{|x| \leq M} e^{r_{0}|K(x)|} d x<\infty$.

Then:

(i) For each closed set $C \in \mathbb{R}$,

$$
\limsup _{n \rightarrow \infty} a(\log \log n)^{-1} \log \left(\operatorname{Pr}\left\{V_{n}(K) \in C\right\}\right) \leq-\inf _{u \in C} I(u, K),
$$

where

$$
I(u, K)=\sup _{r \in \mathbb{R}}\left(r u-f\left(x_{0}\right) \int_{\mathbb{R}^{d}}\left(e^{r K(x)}-1\right) d x\right), \text { for } u \in \mathbb{R} .
$$

(ii) For each open set $G \in \mathbb{R}$,

$$
\liminf _{n \rightarrow \infty} a(\log \log n)^{-1} \log \left(\operatorname{Pr}\left\{V_{n}(K) \in G\right\}\right) \geq-\inf _{u \in G} I(u, K) .
$$


(iii) For each $r>0$, each $u>0$ and each $n \geq 1$,

$$
\operatorname{Pr}\left\{\sum_{j=1}^{n} K\left(b_{n}^{-1}\left(x_{0}-X_{j}\right)\right) \geq u\right\} \leq \exp \left(-u r+n b_{n}^{d} \int_{\mathbb{R}^{d}}\left(e^{r K(x)}-1\right) f\left(x_{0}-b_{n} x\right) d x\right) .
$$

Proof. To show claims (i) and (ii) we apply Theorem II.2 in Ellis (1984) (see also Section 2.3 in Dembo and Zeitouni, 1993). By a change of variables

$$
E\left[e^{r K\left(b_{n}^{-1}\left(x_{0}-X\right)\right)}\right]=1+b_{n}^{d} \int_{\mathbb{R}^{d}}\left(e^{r K(x)}-1\right) f\left(x_{0}-b_{n} x\right) d x,
$$

for $n$ large. So,

$$
\begin{aligned}
a(\log \log n)^{-1} \log \left(E\left[e^{r V_{n}(K)}\right]\right)= & a(\log \log n)^{-1} n \log \left(1+b_{n}^{d} \int_{\mathbb{R}^{d}}\left(e^{r K(x)}-1\right) f\left(x_{0}-b_{n} x\right) d x\right) \\
& \rightarrow f\left(x_{0}\right) \int_{\mathbb{R}^{d}}\left(e^{r K(x)}-1\right) d x,
\end{aligned}
$$

for $r>0$. The rest of the conditions in this theorem are easily checked.

Part (iii) follows from the Cramér method:

$$
\begin{gathered}
\operatorname{Pr}\left\{\sum_{j=1}^{n} K\left(b_{n}^{-1}\left(x_{0}-X_{j}\right)\right) \geq u\right\} \leq e^{-u r} E\left[\exp \left(\sum_{j=1}^{n} r K\left(b_{n}^{-1}\left(x_{0}-X_{j}\right)\right)\right)\right] \\
=e^{-u r}\left(1+b_{n}^{d} \int_{\mathbb{R}^{d}}\left(e^{r K(x)}-1\right) f\left(x_{0}-b_{n} x\right) d x\right)^{n} \\
\leq \exp \left(-u r+n b_{n}^{d} \int_{\mathbb{R}^{d}}\left(e^{r K(x)}-1\right) f\left(x_{0}-b_{n} x\right) d x\right)
\end{gathered}
$$

for $r>0$ (by the Chebyshev inequality).

Let $\left\{N(u): u \in \mathbb{R}^{d}\right\}$ be a multidimensional Poisson process with parameter $f\left(x_{0}\right)$ and let $Z(K)=\int_{\mathbb{R}^{d}} K(x) d N(x)$, for a function $K$ with compact support. Then, the moment generating function of $Z(K)$ is

$$
E\left[e^{r Z(K)}\right]=\exp \left(f\left(x_{0}\right) \int_{\mathbb{R}^{d}}\left(e^{r K(x)}-1\right) d x\right) .
$$

So, the rate function in the Chernoff theorem of $Z(K)$ is $I(u, K)$ (a reference in this topic is Chapter 2 in Dembo and Zeitouni, 1993). In particular, $I(u, K)$ is the Legendre transform of $E\left[e^{r Z(K)}\right]$. Since $E[Z(K)]=f\left(x_{0}\right) \int_{\mathbb{R}^{d}} K(x) d x$, we have that $I(u, K)$ has the following properties:

(i) $I(u, K)$ is a convex function.

(ii) $I(u, K)$ is non-decreasing in $\left[f\left(x_{0}\right) \int_{\mathbb{R}^{d}} K(x) d x, \infty\right)$ and non-increasing in $\left(-\infty, f\left(x_{0}\right) \int_{\mathbb{R}^{d}} K(x) d x\right]$.

(iii) $I(u, K) \geq 0$ and $I\left(f\left(x_{0}\right) \int_{\mathbb{R}^{d}} K(x) d x, K\right)=0$.

(iv) $I(u, f)=\sup _{r \geq 0}\left(r u-f\left(x_{0}\right) \int_{\mathbb{R}^{d}}\left(e^{r K(x)}-1\right) d x\right)$, for $u \geq f\left(x_{0}\right) \int_{\mathbb{R}^{d}} K(x) d x$.

In particular, we have that

$$
\lim _{n \rightarrow \infty} a(\log \log n)^{-1} \log \left(\operatorname{Pr}\left\{V_{n}(K) \geq u\right\}\right)=I(u, K),
$$


for $u \geq f\left(x_{0}\right) \int_{\mathbb{R}^{d}} K(x) d x$.

The following fact is known. Although, we have not found any reference where it is stated exactly in this way.

Lemma 5.2. With the notation the Set-up, let $K$ be a measurable function satisfying (c.3) and (c.4). Then,

$$
\begin{gathered}
\sup \left\{u \geq f\left(x_{0}\right) \int_{\mathbb{R}^{d}} K(x) d x: \sup _{r \in \mathbb{R}}\left(r u-f\left(x_{0}\right) \int_{\mathbb{R}^{d}}\left(e^{r K(x)}-1\right) d x\right) \leq a\right\} \\
\quad=\sup \left\{\int_{\mathbb{R}^{d}} K(x) \alpha(x) d x: \int_{\mathbb{R}^{d}} h\left(\left(f\left(x_{0}\right)\right)^{-1} \alpha(x)\right) d x \leq\left(f\left(x_{0}\right)\right)^{-1} a\right\}
\end{gathered}
$$

where $h(x)=x \log x-x+1$ if $x \geq 0$; and $h(x)=\infty$ if $x<0$.

Moreover, if $r_{K}$ is a any positive number such that $\int_{\mathbb{R}^{d}} h\left(e^{r_{K} K(x)}\right) d x=\left(f\left(x_{0}\right)\right)^{-1}$ a, then the expression (5.6) is $f\left(x_{0}\right) \int_{\mathbb{R}^{d}} K(x) e^{r_{K} K(x)} d x$.

Proof. We will need the following elementary inequality: for each $x \geq 0$ and each $y \in \mathbb{R}$,

$$
x y \leq h(x)+e^{y}-1 .
$$

Let

$$
M_{1}(K, a):=\sup \left\{u \geq f\left(x_{0}\right) \int_{\mathbb{R}^{d}} K(x) d x: \sup _{r \in \mathbb{R}}\left(r u-f\left(x_{0}\right) \int_{\mathbb{R}^{d}}\left(e^{r K(x)}-1\right) d x\right) \leq a\right\}
$$

and let

$$
M_{2}(K, a):=\sup \left\{\int_{\mathbb{R}^{d}} K(x) \alpha(x) d x: \int_{\mathbb{R}^{d}} h\left(\left(f\left(x_{0}\right)\right)^{-1} \alpha(x)\right) d x \leq\left(f\left(x_{0}\right)\right)^{-1} a\right\} .
$$

Suppose that $u<M_{1}$, then there is a real number $r_{0}$ such that

$$
r_{0} u-K\left(x_{0}\right) \int_{\mathbb{R}^{d}}\left(e^{r_{0} K(x)}-1\right) d x=\sup _{r \in \mathbb{R}}\left(r u-f\left(x_{0}\right) \int_{\mathbb{R}^{d}}\left(e^{r K(x)}-1\right) d x\right) \leq a .
$$

Observe that $u=f\left(x_{0}\right) \int_{\mathbb{R}^{d}} K(x) e^{r_{0} K(x)} d x$. Let $\alpha(x)=f\left(x_{0}\right) e^{r_{0} K(x)}$, then

$$
\int_{\mathbb{R}^{d}} h\left(\left(f\left(x_{0}\right)\right)^{-1} \alpha(x)\right) d x=\left(f\left(x_{0}\right)\right)^{-1} r_{0} u-\int_{\mathbb{R}^{d}}\left(e^{r_{0} K(x)}-1\right) d x \leq\left(f\left(x_{0}\right)\right)^{-1} a .
$$

So, $M_{1} \leq M_{2}$. Since $\alpha_{0}(x) \equiv f\left(x_{0}\right)$ satisfies $h\left(\left(f\left(x_{0}\right)\right)^{-1} \alpha_{0}(x)\right) \equiv 0, M_{2} \geq \int_{\mathbb{R}^{d}} K(x) f\left(x_{0}\right) d x$. Suppose that $\int_{\mathbb{R}^{d}} h\left(\left(f\left(x_{0}\right)\right)^{-1} \alpha(x)\right) d x \leq\left(f\left(x_{0}\right)\right)^{-1} a$ and $\int_{\mathbb{R}^{d}} K(x) \alpha(x) d x \geq \int_{\mathbb{R}^{d}} K(x) f\left(x_{0}\right) d x$. By (5.7),

$$
\int_{\mathbb{R}^{d}}\left(r K(x)\left(f\left(x_{0}\right)\right)^{-1} \alpha(x)-e^{r K(x)}+1\right) d x \leq \int_{\mathbb{R}^{d}} h\left(\left(f\left(x_{0}\right)\right)^{-1} \alpha(x)\right) d x \leq\left(f\left(x_{0}\right)\right)^{-1} a
$$

for each $r \in \mathbb{R}$. So, $M_{2} \leq M_{1}$. The rest of the proof follows along similar lines.

Observe that if there exists a $\delta>0$ such that $\lambda\left\{x \in \mathbb{R}^{d}: f(x) \geq \delta\right\} \geq \delta$, then $\psi(r)=$ $\int_{\mathbb{R}^{d}} h\left(e^{r K(x)}\right) d x$ is a continuous function with $\psi(0)=0$ and $\lim _{r \rightarrow \infty} \psi(r)=\infty$. So, there exists a positive number $r_{K}$ such that $\psi\left(r_{K}\right)=\left(f\left(x_{0}\right)\right)^{-1} a$. 
We will denote

$$
M(K, a):=\sup \left\{\int_{\mathbb{R}^{d}} K(x) \alpha(x) d x: \int_{\mathbb{R}^{d}} h\left(\left(f\left(x_{0}\right)\right)^{-1} \alpha(x)\right) d x \leq\left(f\left(x_{0}\right)\right)^{-1} a\right\} .
$$

We will consider two cases of L.I.L. for a processes in the Poisson domain because when the function is non-negative the assumptions are weaker.

TheOrem 5.3. With the notation in the Set-up, assume (c.1) and (c.2). Let $K$ be a nonnegative function satisfying (c.3), (c.4),

$\lim _{\delta \rightarrow \infty} \limsup _{n \rightarrow 0}(\log \log n)^{-1} E\left[\sup _{1-\delta \leq u \leq 1}\left|\sum_{j=1}^{n}\left(K\left(u^{-1} b_{n}^{-1}\left(x_{0}-X_{j}\right)\right)-K\left(b_{n}^{-1}\left(x_{0}-X_{j}\right)\right)\right)\right|\right] \longrightarrow 0$.

(c.6) For each $r>0$, there exists a $\delta>0$ such that

$$
\int_{\mathbb{R}^{d}} \exp \left(r \sup _{1-\delta \leq u \leq 1}\left|K\left(u^{-1} x\right)-K(x)\right|\right) d x<\infty .
$$

Then,

$$
\limsup _{n \rightarrow \infty} a(\log \log n)^{-1} \sum_{j=1}^{n} K\left(b_{n}^{-1}\left(x_{0}-X_{j}\right)\right)=M(K, a) \text { a.s. }
$$

Proof. Let $\delta>0$ and let $\gamma>1$. For $\left[\gamma^{j}\right] \leq n \leq\left[\gamma^{j+1}\right]$, we have that

$$
\begin{gathered}
V_{n}(K) \leq a\left(\log \log \left[\gamma^{j}\right]\right)^{-1} \sum_{i=1}^{\left[\gamma^{j+1}\right]} K\left(b_{n}^{-1}\left(x_{0}-X_{i}\right)\right) \\
\leq a\left(\log \log \left[\gamma^{j}\right]\right)^{-1} \sum_{i=1}^{\left[\gamma^{j+1}\right]} K\left(b_{\left[\gamma^{j}\right]}^{-1}\left(x_{0}-X_{i}\right)\right) \\
+a\left(\log \log \left[\gamma^{j}\right]\right)^{-1} \sup _{1-\delta \leq u \leq 1}\left|\sum_{i=1}^{\left[\gamma^{j+1}\right]}\left(K\left(u^{-1} b_{\left[\gamma^{j}\right]}^{-1}\left(x_{0}-X_{i}\right)\right)-K\left(b_{\left[\gamma^{j}\right]}^{-1}\left(x_{0}-X_{i}\right)\right)\right)\right|,
\end{gathered}
$$

if $\gamma$ is close enough to one. Given $\eta>M(f, a)$, there exists a $r>0$ such that $r \eta-f\left(x_{0}\right) \int_{\mathbb{R}^{d}}\left(e^{r K(x)}-1\right) d x>a$. So, by Lemma 1

$$
\operatorname{Pr}\left\{a\left(\log \log \left[\gamma^{j}\right]\right)^{-1} \sum_{i=1}^{\left[\gamma^{j+1}\right]} K\left(b_{\left[\gamma^{j}\right]}^{-1}\left(x_{0}-X_{i}\right)\right) \geq \gamma \eta\right\} \leq j^{-s}
$$

for $j$ large enough and some $s>1$. Hence

$$
\limsup _{j \rightarrow \infty} a\left(\log \log \left[\gamma^{j}\right]\right)^{-1} \sum_{i=1}^{\left[\gamma^{j+1}\right]} K\left(b_{\left[\gamma^{j}\right]}^{-1}\left(x_{0}-X_{i}\right)\right) \leq M(K, a) \quad \text { a.s. }
$$


We also have that

$$
\begin{gathered}
a(\log j)^{-1} \sup _{1-\delta \leq u \leq 1}\left|\sum_{i=1}^{\left[\gamma^{j+1}\right]}\left(K\left(u^{-1} b_{\left[\gamma^{j}\right]}^{-1}\left(x_{0}-X_{i}\right)\right)-K\left(b_{\left[\gamma^{j}\right]}^{-1}\left(x_{0}-X_{i}\right)\right)\right)\right| \\
\leq a(\log j)^{-1} \sum_{i=1}^{\left[\gamma^{j+1}\right]} \bar{G}_{\delta}\left(b_{\left[\gamma^{j}\right]}^{-1}\left(x_{0}-X_{i}\right)\right) I_{\bar{G}_{\delta}\left(b_{\left[\gamma^{j}\right]}^{-1}\left(x_{0}-X_{i}\right)\right)>M} \\
+a(\log j)^{-1}\left[\gamma^{j+1}\right] \sup _{1-\delta \leq u \leq 1}\left|E\left[\left(K\left(u^{-1} b_{\left[\gamma^{j}\right]}^{-1}\left(x_{0}-X\right)\right)-K\left(b_{\left[\gamma^{j}\right]}^{-1}\left(x_{0}-X\right)\right)\right) I_{\bar{G}_{\delta}\left(b_{\left[\gamma^{j}\right]}^{-1}\left(x_{0}-X\right)\right) \leq M}\right]\right| \\
+a(\log j)^{-1} \sup _{1-\delta \leq u \leq 1} \mid \sum_{i=1}^{\left[\gamma^{j+1}\right]}\left(K\left(u^{-1} b_{\left[\gamma^{j}\right]}^{-1}\left(x_{0}-X_{i}\right)\right)-K\left(b_{\left[\gamma^{j}\right]}^{-1}\left(x_{0}-X_{i}\right)\right)\right) I_{\bar{G}_{\delta}\left(b_{\left[\gamma^{j}\right]}^{-1}\left(x_{0}-X_{i}\right)\right) \leq M} \\
\left.-E\left[K\left(u^{-1} b_{\left[\gamma^{j}\right]}^{-1}\left(x_{0}-X_{i}\right)\right) I_{\bar{G}_{\delta}\left(b_{\left[\gamma^{j}\right]}^{-1}\left(x_{0}-X_{i}\right)\right) \leq M}-K\left(b_{\left[\gamma^{j}\right]}^{-1}\left(x_{0}-X_{i}\right)\right) I_{\bar{G}_{\delta}\left(b_{\left[\gamma^{j}\right]}^{-1}\left(x_{0}-X_{i}\right)\right) \leq M}\right]\right) \mid \\
=: I_{M, \delta, j}+I I_{M, \delta, j}+I I I_{M, \delta, j},
\end{gathered}
$$

where $\bar{G}_{\delta}(x):=\sup _{1-\delta \leq u \leq 1}\left|K\left(u^{-1} x\right)-K(x)\right|$. Given $\eta>0$, let $r=3 \eta^{-1}$. Then, there exists a $\delta>0$ such that $\int\left(e^{r \bar{G}_{\delta}(\bar{x})}-1\right) d x<\infty$. So, if $M$ large enough,

$$
\gamma \int_{\mathbb{R}^{d}}\left(e^{r \overline{G_{\delta}}(x) I_{\bar{G}_{\delta}(x) \geq M}}-1\right) d x \leq 1 .
$$

By Lemma 5.1,

$$
\begin{gathered}
\operatorname{Pr}\left\{\sum_{i=1}^{\left[\gamma^{j+1}\right]} \bar{G}_{\delta}\left(b_{\left[\gamma^{j}\right]}^{-1}\left(x_{0}-X_{i}\right)\right) I_{\bar{G}_{\delta}\left(b_{\left[\gamma^{j}\right]}^{-1}\right.}\left(x_{0}-X_{i}\right)\right) \geq M \\
\leq \eta \log j\} \\
\leq \exp \left(-r \eta \log j+\left[\gamma^{j+1}\right] b_{\left[\gamma^{j}\right]} \int_{\mathbb{R}^{d}}\left(e^{r \bar{G}_{\delta}(x)}-1\right) I_{\bar{G}_{\delta}(x) \geq M} f\left(x_{0}-b_{\left[\gamma^{j}\right]} x\right) d x\right) \leq j^{-2},
\end{gathered}
$$

for $j$ large. So,

$$
\lim _{M \rightarrow \infty} \limsup _{\delta \rightarrow 0} \limsup _{n \rightarrow \infty} I_{M, \delta, j}=0 \text { a.s. }
$$

By (b.2) and the Lusin theorem

$$
I I_{M, \delta, j} \leq 0(1) \sup _{1-\delta \leq u \leq 1} \int_{\mathbb{R}^{d}}\left|K\left(u^{-1} x\right)-K(x)\right| d x \rightarrow 0 .
$$

By Theorem 2.4, for each $M<\infty$ there exist a $\delta>0$, such that

$$
\sum_{j=1}^{\infty} \operatorname{Pr}\left\{I I I_{M, \delta, j} \geq \eta\right\}<\infty .
$$

Observe that we need that

$$
\lim _{M \rightarrow 0} \limsup _{\delta \rightarrow 0} \limsup _{j \rightarrow \infty} E\left[I I I_{M, \delta, j}\right]=0 .
$$


This condition follows from (c.5). So,

$$
\lim _{M \rightarrow 0} \limsup _{\delta \rightarrow 0} \limsup _{n \rightarrow \infty} I I I_{M, \delta, j}=0 \text { a.s. }
$$

for each $M<\infty$. From (5.11)-(5.16)

$$
\limsup _{n \rightarrow \infty} V_{n}(K) \leq M(K, a) \text { a.s. }
$$

By the second lemma of Borel-Cantelli and Lemmas 1 and 2

$$
\limsup _{j \rightarrow \infty} a(\log j)^{-1} \sum_{k=\left[\gamma^{j-1}\right]+1}^{\left[\gamma^{j}\right]} K\left(b_{\left[\gamma^{j}\right]}^{-1}\left(X_{k}-x_{0}\right)\right)=(\gamma-1) \gamma^{-1} M\left(K, a \gamma(\gamma-1)^{-1}\right) \text { a.s. }
$$

So,

$$
\limsup _{j \rightarrow \infty} V_{\left[\gamma^{j}\right]}(f) \geq(\gamma-1) \gamma^{-1} M\left(K, a \gamma(\gamma-1)^{-1}\right) \text { a.s. }
$$

Since $(\gamma-1) \gamma^{-1} M\left(K, a \gamma(\gamma-1)^{-1}\right) \rightarrow M(K, a)$ as $\gamma \rightarrow \infty$, the result follows.

The last theorem is a generalization of Theorem 8 in Deheuvels and Mason (1991) to the multivariate and non-uniform case, under weaker hypotheses. Observe that

$$
\begin{gathered}
(\log \log n)^{-1} E\left[\sup _{1-\delta \leq u \leq 1} \mid \sum_{j=1}^{n}\left(K\left(u^{-1} b_{n}^{-1}\left(x_{0}-X_{j}\right)\right)-K\left(b_{n}^{-1}\left(x_{0}-X_{j}\right)\right) \mid\right]\right. \\
\leq(\log \log n)^{-1} n E\left[\sup _{1-\delta \leq u \leq 1}\left|K\left(u^{-1} b_{n}^{-1}\left(x_{0}-X\right)\right)-K\left(b_{n}^{-1}\left(x_{0}-X\right)\right)\right|\right] \\
=O(1) \int_{\mathbb{R}^{d}} \sup _{1-\delta \leq u \leq 1}\left|K\left(u^{-1} x\right)-K(x)\right| d x .
\end{gathered}
$$

So, condition (c.5) follows from the condition

$$
\lim _{\delta \rightarrow 0} \int_{\mathbb{R}^{d}} \sup _{1-\delta \leq u \leq 1}\left|K\left(u^{-1} x\right)-K(x)\right| d x=0 .
$$

By a previous argument, we have that if the class $\left\{K\left(u^{-1} x\right): 0 \leq u \leq 1\right\}$ is a VC subgraph class and

$$
\lim _{\delta \rightarrow 0+} \int_{\mathbb{R}^{d}} \sup _{1-\delta \leq u \leq 1}\left|K\left(u^{-1} x\right)-K(x)\right| d x<\infty
$$

then (c.5) follows.

THEOREm 5.4. With the notation in the Set-up, assume (c.1) and (c.2). Let $K$ be a function satisfying (c.3), (c.5), (c.6) and

(c.7) For each $0<r<\infty, \int_{|x| \leq M} e^{r|K(x)|} d x<\infty$.

Then,

$$
\limsup _{n \rightarrow \infty} a(\log \log n)^{-1} \sum_{j=1}^{n} K\left(b_{n}^{-1}\left(x_{0}-X_{j}\right)\right)=M(K, a) \text { a.s. }
$$


Proof. By Lemma 5.1 and the lemma of Borel-Cantelli

$$
\limsup _{j \rightarrow \infty} V_{\left[\gamma^{j]}\right.}(K) \leq M(K, a) \text { a.s. }
$$

We claim that

$$
\limsup _{\gamma \rightarrow 1+} \limsup _{j \rightarrow \infty} \sup _{\left[\gamma^{j}\right] \leq n \leq\left[\gamma^{j+1}\right]}\left|V_{\left[\gamma^{j}\right]}(K)-V_{n}(K)\right|=0 \text { a.s. }
$$

We have that

$$
\begin{gathered}
V_{\left[\gamma^{j}\right]}(K)-V_{n}(K)=a(\log \log n)^{-1} \sum_{i=1}^{n}\left(K\left(b_{n}^{-1}\left(x_{0}-X_{i}\right)\right)-K\left(b_{\left[\gamma^{j}\right]}^{-1}\left(x_{0}-X_{i}\right)\right)\right. \\
+a(\log \log n)^{-1} \sum_{i=\left[\gamma^{j}\right]+1}^{n} K\left(b_{\left[\gamma^{j}\right]}^{-1}\left(x_{0}-X_{i}\right)\right) \\
+a\left((\log \log n)^{-1}-\left(\log \log \left[\gamma^{j}\right]\right)^{-1}\right) \sum_{i=\left[\gamma^{j}\right]+1}^{n} K\left(b_{\left[\gamma^{j}\right]}^{-1}\left(x_{0}-X_{i}\right)\right) \\
=: I_{n}+I I_{n}+I I I_{n} .
\end{gathered}
$$

By the argument in Theorem 5.3

$$
\limsup _{\gamma \rightarrow 1+} \limsup _{j \rightarrow \infty} \sup _{\left[\gamma^{j}\right] \leq n \leq\left[\gamma^{j+1}\right]}\left|I_{n}\right|=0 \quad \text { a.s. }
$$

We have that

$$
\sup _{\left[\gamma^{j}\right] \leq n \leq\left[\gamma^{j+1}\right]}\left|I I_{n}\right| \leq a\left(\log \log \left[\gamma^{j}\right]\right)^{-1} \sum_{i=\left[\gamma^{j}\right]+1}^{\left[\gamma^{j+1}\right]}\left|K\left(b_{\left[\gamma^{j}\right]}^{-1}\left(x_{0}-X_{i}\right)\right)\right|
$$

By Lemma 5.1

$$
\begin{gathered}
\operatorname{Pr}\left\{\sum_{i=\left[\gamma^{j}\right]+1}^{\left[\gamma^{j+1}\right]}\left|K\left(b_{\left[\gamma^{j}\right]}^{-1}\left(x_{0}-X_{i}\right)\right)\right| \geq \eta \log j\right\} \\
\leq \exp \left(-r \eta \log j+\left(\left[\gamma^{j+1}\right]-\left[\gamma^{j}\right]\right) b_{\left[\gamma^{j}\right]} E\left[e^{r|K(X)|}-1\right]\right) \leq j^{-2},
\end{gathered}
$$

if $r \eta>3$ and $\gamma$ is close enough to one. The term $I I I_{n}$ can be dealt with similarly. By the second lemma of Borel-Cantelli and Lemma 1

$$
\limsup _{j \rightarrow \infty} a(\log j)^{-1} \sum_{k=\left[\gamma^{j-1}\right]+1}^{\left[\gamma^{j}\right]} K\left(b_{\left[\gamma^{j}\right]}^{-1}\left(x_{0}-X_{k}\right)\right)=(\gamma-1) \gamma^{-1} M\left(K, a \gamma(\gamma-1)^{-1}\right) \text { a.s. }
$$

and

$$
\limsup _{j \rightarrow \infty} a(\log j)^{-1} \sum_{k=1}^{\left[\gamma^{j-1}\right]} K\left(b_{\left[\gamma^{j}\right]}^{-1}\left(x_{0}-X_{k}\right)\right) \leq \gamma^{-1} M(K, a \gamma) \quad \text { a.s. }
$$

So,

$$
\limsup _{n \rightarrow \infty} V_{n}(K) \geq(\gamma-1) \gamma^{-1} M\left(K, a \gamma(\gamma-1)^{-1}\right)-\gamma^{-1} M(K, a \gamma) \text { a.s. }
$$


We have that $(\gamma-1) \gamma^{-1} M\left(K, a \gamma(\gamma-1)^{-1}\right) \rightarrow M(K, a)$ as $\gamma \rightarrow \infty$. Fix $r>0$,

$$
r\left(2 a \gamma r^{-1}\right)-\int_{\mathbb{R} d}\left(e^{r K(x)}-1\right) d x>a \gamma
$$

for $\gamma$ large. So, $M(K, a \gamma) \leq 2 a \gamma r^{-1}$ for $\gamma$ large. Hence, $\gamma^{-1} M(K, a \gamma) \rightarrow 0$ as $\gamma \rightarrow \infty$. Therefore, the result follows.

Next, we will consider the L.I.L. uniformly over a class of functions.

It is easy to see that the proof of Lemma 2 in Finkelstein (1971) applies to this situation, giving the following:

LEMMA 5.5. With the notation in the Set-up, assume (c.1) and (c.2). Let $K_{1}, \ldots, K_{p}$ be measurable functions in $\mathbb{R}^{d}$ satisfying (c.3) and (c.5)-(c.7). Then, with probability one,

$$
\left(V_{n}\left(K_{1}\right), \ldots, V_{n}\left(K_{p}\right)\right)
$$

is relatively compact and its limit set is

$$
\left\{\left(\int_{\mathbb{R}^{d}} K_{1}(x) \alpha(x) d x, \ldots, \int_{\mathbb{R}^{d}} K_{p}(x) \alpha(x) d x\right): \int_{\mathbb{R}^{d}} h\left(\left(f\left(x_{0}\right)\right)^{-1} \alpha(x)\right) d x \leq\left(f\left(x_{0}\right)\right)^{-1} a\right\} .
$$

We also need the following:

LEMMA 5.6. Let $K$ be a measurable function on $\mathbb{R}^{d}$ such that $K(x)=0$, if $|x|>M$, and let $0<a<\infty$. We define

$$
\|K\|_{\mathrm{h}}=\sup \left\{\left|\int_{\mathbb{R}^{d}} K(x) \alpha(x) d x\right|: \int_{|x| \leq M} h\left(\left(f\left(x_{0}\right)\right)^{-1} \alpha(x)\right) d x \leq a\left(f\left(x_{0}\right)\right)^{-1}\right\} .
$$

\section{Then}

(i) If $a \geq K\left(x_{0}\right) \lambda\{|x| \leq M\}, \int_{\mathbb{R}^{d}}|K(x)| d x \leq 2\left(f\left(x_{0}\right)\right)^{-1}\|K\|_{\mathrm{h}}$.

(ii) If $f\left(x_{0}\right) \lambda\{|x| \leq M\}>a>0, \int_{|x| \leq M}|K(x)| d x \leq 2\left(f\left(x_{0}\right)\right)^{-1}(1-z)^{-1}\|K\|_{\mathrm{h}}$, where $z$ is the number such that $0<z<1$ and $h(z)=a\left(f\left(x_{0}\right) \lambda\{|x| \leq M\}\right)^{-1}$.

(iii) For any $r>0$,

$$
\|K\|_{\mathrm{h}} \leq f\left(x_{0}\right) t^{-1} a+f\left(x_{0}\right)\left(\int_{|x| \leq M} K^{2}(x) d x\right)^{1 / 2}\left(\int_{|x| \leq M} e^{2 r|K(x)|} d x\right)^{1 / 2} .
$$

Proof. To prove (i), for $C \subset\left\{x \in \mathbb{R}^{d}:|x| \leq M\right\}$, take $\alpha=f\left(x_{0}\right) I_{C^{c}}$. Since $\int_{|x| \leq M} h\left(\left(f\left(x_{0}\right)\right)^{-1} \alpha(x)\right) d x=\lambda(C) \leq a\left(f\left(x_{0}\right)\right)^{-1},\|K(\cdot)\|_{\mathrm{h}} \geq f\left(x_{0}\right)\left|\int_{C} K(x) d x\right|$. Applying this relation for $C=\{K(x)>0\}$ and $C=\{K(x)<0\}$, we get (i). To prove (ii), for $C \subset\left\{x \in \mathbb{R}^{d}\right.$ : $|x| \leq M\}$ take $\alpha=f\left(x_{0}\right) z+f\left(x_{0}\right)(1-z) I_{C}$ and proceed as before.

As to (iii) if $\int_{|x| \leq M} h\left(\left(f\left(x_{0}\right)\right)^{-1} \alpha(x)\right) d x \leq a\left(f\left(x_{0}\right)\right)^{-1}$, by $(5.7)$

$$
\left(f\left(x_{0}\right)\right)^{-1}\left|\int_{|x| \leq M} K(x) \alpha(x) d x\right| \leq t^{-1}\left|\int_{|x| \leq M}\left(e^{t|K(x)|}-1+h\left(\left(f\left(x_{0}\right)\right)^{-1} \alpha(x)\right)\right) d x\right|
$$




$$
\leq r^{-1} a+\int_{|x| \leq M}|K(x)| e^{r|K(x)|} d x \leq r^{-1} a+\left(\int_{|x| \leq M} K^{2}(x) d x\right)^{1 / 2}\left(\int_{|x| \leq M} e^{2 r|K(x)|} d x\right)^{1 / 2} .
$$

So, the result follows.

Finally, we consider the L.I.L. uniformly over a class of functions.

TheOREM 5.7. Let $\left\{X_{j}\right\}_{j=1}^{\infty}$ be a sequence of i.i.d.r.v.'s with values on $\mathbb{R}^{d}$, let $x_{0} \in \mathbb{R}^{d}$, let $\left\{K_{t}: t \in T\right\}$ be a collection of measurable functions in $\mathbb{R}^{d}$ and let $\left\{b_{n}\right\}$ be a sequence of positive numbers. Suppose that:

(c.1) There exists a positive number $a$, such that $n^{-1} b_{n}^{-d} \log \log n \rightarrow a$.

(c.2) $X$ has a density $f$ in a neighborhood of $x_{0}$, which is continuous and positive at $x_{0}$.

(c.3) For each $t \in T, K_{t}(x)=0$ if $|x|>M$.

$$
\lim _{\delta \rightarrow \infty} \limsup _{n \rightarrow 0} E\left[\sup _{1-\delta \leq u \leq 1} \sup _{t \in T} \mid V_{n}\left(K_{t}\left(u^{-1} \cdot\right)-E\left[V_{n}\left(K_{t}\left(u^{-1} \cdot\right)\right)\right] \mid\right]=0 .\right.
$$

(c.6) For each $r>0$ and each $t \in T$, there exists a $\delta>0$ such that

$$
\int_{\mathbb{R}^{d}} \exp \left(r \sup _{1-\delta \leq u \leq 1}\left|K\left(u^{-1} x\right)-K(x)\right|\right) d x<\infty .
$$

(c.7) For each $0<r<\infty$,

$$
\int_{|x| \leq M} \exp \left(r \sup _{t \in T}\left|K_{t}(x)\right|\right) d x<\infty .
$$

(c.8) $(T, \rho)$ is totally bounded, where $\rho(s, t)=\int_{\mathbb{R}^{d}}\left|K_{s}(x)-K_{t}(x)\right| d x$.

Then, with probability one,

$$
\left\{a(\log \log n)^{-1} \sum_{j=1}^{n} K_{t}\left(b_{n}^{-1}\left(x_{0}-X_{j}\right)\right): t \in T\right\}
$$

is relatively compact and its limit set is

$$
\left\{\left(\int_{\mathbb{R}^{d}} K_{t}(x) \alpha(x) d x\right)_{t \in T}: \int_{\mathbb{R}^{d}} h\left(\left(f\left(x_{0}\right)\right)^{-1} \alpha(x)\right) d x \leq\left(f\left(x_{0}\right)\right)^{-1} a\right\} .
$$

Proof. We apply Lemma 2.1. Lemma 5.5 implies the L.I.L. for the finite dimensional distributions. By the argument used in Theorem 3 to deal with in $I_{M, \delta, j}$,

$$
\lim _{M \rightarrow \infty} \limsup _{n \rightarrow \infty} \sup _{t \in T}\left|V_{n}\left(K_{t} I_{G \geq M}\right)\right|=0 \text { a.s., }
$$

where $G(x)=\sup _{t \in T}\left|K_{t}(x)\right|$. By Theorem 2.4 (condition (c.2) in Remark 2.5 is satisfied)

$$
\lim _{\delta \rightarrow 0} \limsup _{n \rightarrow \infty} \sup _{\rho\left(t_{1}, t_{2}\right) \leq \delta} \mid\left(V_{n}\left(\left(K_{t_{1}}-K_{t_{2}}\right) I_{G \leq M}\right)-E\left[V_{n}\left(\left(K_{t_{1}}-K_{t_{2}}\right) I_{G \leq M}\right)\right] \mid=0\right. \text { a.s. }
$$


for each $M<\infty$. Obviously

$$
\lim _{\delta \rightarrow 0} \limsup _{n \rightarrow \infty} \sup _{\rho\left(t_{1}, t_{2}\right) \leq \delta}\left|E\left[V_{n}\left(\left(K_{t_{1}}-K_{t_{2}}\right) I_{G \leq M}\right)\right]\right|=0,
$$

for each $M<\infty$. So, the claim follows.

If $u t \in T$, for each $0 \leq u \leq 1$ and each $t \in T$, and conditions (c.1), (c.2), (c.3) and (c.7) in the previous theorem are satisfied, then Lemma 2.2 gives that $\left\{V_{n}\left(K_{t}\right): t \in T\right\}$ satisfies the compact L.I.L. if and only if $(T, \rho)$ is totally bounded and $\sup _{t \in T}\left|V_{n}\left(K_{t}\right)\right| \stackrel{\mathrm{Pr}}{\longrightarrow} 0$.

In the case that the functions are nonnegative, satisfying (c.4) and (c.6), condition (c.7) can be relaxed to

(c.7)" For each $0<r<\infty$, there exists a $\delta>0$ such that

$$
\int_{|x| \leq M} \exp \left(r \sup _{\rho(s, t) \leq \delta}\left|K_{t}(x)\right|\right) d x<\infty .
$$

By the Remark 1.5, if $\left\{K_{t}\left(u^{-1} x\right): 0 \leq u \leq 1, t \in T\right\}$ is a VC subgraph class of functions such that

$$
\lim _{\delta \rightarrow 0+} \int_{\mathbb{R}^{d}} \sup _{1-\delta \leq u \leq 1} \sup _{t \in T}\left|K_{t}\left(u^{-1} x\right)\right| d x<\infty,
$$

then conditions (c.5) and (c.8) in Theorem 5.7 are satisfied. As to bracketing, we have the following:

Proposition 5.8. With the previous notation, assume conditions (c.1), (c.2), (c.3), (c.9) $\int_{\mathbb{R}^{d}}\left|K_{t}(x)\right| d x<\infty$, for each $t \in T$.

(c.10) $N_{[]}^{(1)}(\tau, T, \lambda)<\infty$, for each $\tau<\infty$.

Then,

$$
\sup _{t \in T}\left|V_{n}\left(K_{t}\right)-E\left[V_{n}\left(K_{t}\right)\right]\right| \stackrel{\text { Pr }}{\longrightarrow} 0 .
$$

Proof. $c$ will denote a universal constant which may varies from line to line. We claim that if $f$ satisfied (c.9), then

$$
\mid V_{n}\left(K_{t}\right)-E\left[V_{n}\left(K_{t}\right]\right) \stackrel{\operatorname{Pr}}{\longrightarrow} 0
$$

Given $\epsilon>0$,

$$
E\left[\left|V_{n}\left(K_{t} I_{\left|K_{t}(x)\right|>b}\right)-E\left[V_{n}\left(K_{t} I_{\left|K_{t}(x)\right|>b}\right)\right]\right| \leq c \int_{\mathbb{R}^{d}}\left|K_{t}(x)\right| I_{\left|K_{t}(x)\right|>b} d x \leq \epsilon,\right.
$$

where and $b$ is a large enough constant. We also have that

$$
\operatorname{Var}\left(V_{n}\left(K_{t} I_{\left|K_{t}(x)\right| \leq b}\right)\right) \leq c(\log \log n)^{-1} \int_{\mathbb{R}^{d}} K_{t}^{2}(x) I_{\left|K_{t}(x)\right| \leq b} d x,
$$

which goes to zero. So, (5.26) holds. 
Given $\tau>0$, there are $K_{t_{1}}, \ldots, K_{t_{m}} \in \mathcal{F}$ and functions $\Delta_{1}, \ldots, \Delta_{m}$ such that $\int_{\mathbb{R}^{d}} \Delta_{k}(x) d x \leq \tau$ for each $1 \leq k \leq m$, and for each $t \in T$, there exists a $1 \leq k \leq m$ such that $\left|K_{t}(x)-K_{t_{k}}(x)\right| \leq$ $\Delta_{k}(x)$, for each $x \in \mathbb{R}^{d}$. Hence

$$
\begin{aligned}
& \sup _{t \in T}\left|V_{n}(K)-E\left[V_{n}\left(K_{t}\right)\right]\right| \leq \sup _{1 \leq k \leq m}\left|V_{n}\left(K_{t_{k}}\right)-E\left[V_{n}\left(K_{t_{k}}\right)\right]\right| \\
& \quad+\sup _{1 \leq k \leq m}\left|V_{n}\left(\Delta_{k}\right)-E\left[V_{n}\left(\Delta_{k}\right)\right]\right|+2 \sup _{1 \leq k \leq m}\left|E\left[V_{n}\left(\Delta_{k}\right)\right]\right| .
\end{aligned}
$$

We have that $\left|E\left[V_{n}\left(\Delta_{k}\right)\right]\right| \leq 2 f\left(x_{0}\right) \int_{\mathbb{R}^{d}}\left|\Delta_{k}(x)\right| d x$, for $n$ large enough. So,

$$
\begin{gathered}
\operatorname{Pr}\left\{\sup _{t \in T}\left|V_{n}\left(K_{t}\right)-E\left[V_{n}\left(K_{t}\right)\right]\right| \geq 2\left(f\left(x_{0}\right)+1\right) \eta\right\} \\
\leq \operatorname{Pr}\left\{\sup _{1 \leq k \leq m}\left|V_{n}\left(K_{t_{k}}\right)-E\left[V_{n}\left(K_{t_{k}}\right)\right]\right| \geq \eta\right\}+\operatorname{Pr}\left\{\sup _{1 \leq k \leq m}\left|V_{n}\left(\Delta_{k}\right)-E\left[V_{n}\left(\Delta_{k}\right)\right]\right| \geq \eta\right\},
\end{gathered}
$$

which goes to zero.

So, if there exists a $\delta>0$ such that $N_{[]}^{(1)}\left(\tau, T_{\delta}, \lambda\right)<\infty$, for each $\tau<\infty$, where $\left\{K_{t}\left(u^{-1} x\right)\right.$ : $1-\delta \leq u \leq 1, t \in T\}$, then (c.5) in Theorem 5.7 holds.

Condition (c.5) is also satisfied for VC subgraph classes of functions. Definition and main properties of VC classes of sets can be found in Chapters 9 and 11 in Dudley (1984). Given a function $f: S \rightarrow \mathbb{R}$, the subgraph of $f$ is the set $\{(x, t) \in S \times \mathbb{R}: 0 \leq t \leq f(x)$ or $f(x) \leq t \leq 0\}$. A class of functions $\mathcal{F}$ is a VC-subgraph class if the collection of subgraphs of $\mathcal{F}$ is a VC class. It is well known that for any $\mathrm{VC}$ subgraph class of functions and any $n$,

$$
E\left[\sup _{f \in \mathcal{F}}\left|\sum_{j=1}^{n} \epsilon_{j} f\left(X_{j}\right)\right|\right] \leq c E\left[\left|\sum_{j=1}^{n} \epsilon_{j} \sup _{f \in \mathcal{F}}\right| f\left(X_{j}\right)||\right],
$$

where $\left\{\epsilon_{j}\right\}$ is a sequence of Rademacher r.v.'s, independent of the sequence $\left\{X_{j}\right\}$, and $c$ is universal constant depending only on the class $\mathcal{F}$.

Proposition 5.9. With the notation in Theorem 5.7, assume conditions (c.1)-(c.3) in this theorem. Suppose also that there exists a $0<\delta_{0}<1$ such that:

(i) $\left\{K_{t}\left(u^{-1} x\right): 1-\delta_{0} \leq u \leq 1, t \in T\right\}$ is a VC subgraph class of functions.

(ii)

$$
\int \sup _{1-\delta_{0} \leq u \leq 1} \sup _{t \in T}\left|K_{t}\left(u^{-1} x\right)\right| d x<\infty .
$$

Then,

$$
\lim _{\delta \rightarrow \infty} \limsup _{n \rightarrow 0} E\left[\sup _{1-\delta \leq u \leq 1, t \in T} \mid V_{n}\left(K_{t}\left(u^{-1} \cdot\right)-E\left[V_{n}\left(K_{t}\left(u^{-1} \cdot\right)\right] \mid\right]=0 .\right.\right.
$$

Proof. Let $\tau>0$. By symmetrization and (5.27),

$$
E\left[\sup _{1-\delta \leq u \leq 1, t \in T}\left|V_{n}\left(K_{t}, u^{-1}\right)-E\left[V_{n}\left(K_{t}, u^{-1}\right)\right]\right|\right]
$$




$$
\begin{gathered}
\leq 2 E\left[\sup _{1-\delta \leq u \leq 1, t \in T}(\log \log n)^{-1} \mid \sum_{j=1}^{n} \epsilon_{j} K_{t}\left(u^{-1} b_{n}^{-1}\left(x_{0}-X_{j}\right) \mid\right]\right. \\
\leq c E\left[(\log \log n)^{-1}\left|\sum_{j=1}^{n} \epsilon_{j} \sup _{1-\delta \leq u \leq 1, t \in T}\right| K_{t}\left(u^{-1} b_{n}^{-1}\left(x_{0}-X_{j}\right)||\right]\right. \\
\leq c E\left[(\log \log n)^{-1}\left|\sum_{j=1}^{n} \epsilon_{j} \sup _{1-\delta \leq u \leq 1, t \in T}\right| K_{t}\left(u^{-1} b_{n}^{-1}\left(x_{0}-X_{j}\right)\left|I_{\sup _{1-\delta \leq u \leq 1} \sup _{t \in T}\left|K_{t}\left(u^{-1} b_{n}^{-1}\left(x_{0}-X_{j}\right)\right)\right| \leq \tau}\right|\right]\right. \\
+c E\left[(\log \log n)^{-1}\left|\sum_{j=1}^{n} \epsilon_{j} \sup _{1-\delta \leq u \leq 1, t \in T}\right| K_{t}\left(u^{-1} b_{n}^{-1}\left(x_{0}-X_{j}\right)\right)\left|I_{\sup _{1-\delta \leq u \leq 1, t \in T} \mid K_{t}\left(u^{-1} b_{n}^{-1}\left(x_{0}-X_{j}\right) \mid>\tau\right.}\right|\right] \\
\leq c(\log \log n)^{-1}\left(n E\left[\sup _{1-\delta \leq u \leq 1, t \in T} \mid K_{t}\left(\left.u^{-1} b_{n}^{-1}\left(x_{0}-X_{j}\right)\right|^{2} I_{\sup _{1-\delta \leq u \leq 1, t \in T} \mid K_{t}\left(u^{-1} b_{n}^{-1}\left(x_{0}-X_{j}\right) \mid \leq \tau\right.}\right]\right)^{1 / 2}\right. \\
+c(\log \log n)^{-1} E\left[n \sup _{1-\delta \leq u \leq 1, t \in T} \mid K_{t}\left(u^{-1} b_{n}^{-1}\left(x_{0}-X_{j}\right) \mid I_{\sup _{1-\delta \leq u \leq 1, t \in T} \mid K_{t}\left(u^{-1} b_{n}^{-1}\left(x_{0}-X_{j}\right) \mid>\tau\right.}\right]\right. \\
\leq c(\log \log n)^{-1 / 2}+c \int \sup _{1-\delta \leq u \leq 1, t \in T}\left|K_{t}\left(u^{-1} x\right)\right| I_{\sup _{1-\delta \leq u \leq 1, t \in T}\left|K_{t}\left(u^{-1} x\right)\right|>\tau} d x
\end{gathered}
$$

So, the claim follows.

\section{References}

[1] Alexander, K. S. (1987). Central limit theorems for stochastic processes under random entropy conditions. Probab. Theor. Rel. Fields 75 351-378.

[2] Arcones, M. A. (1994). Some strong limit theorems for M-estimators. Stoch. Proc. Appl. 53 241-268.

[3] Arcones, M. A. (1995a). Weak convergence for the row sums of a triangular array of empirical processes indexed by a manageable triangular array of functions.

[4] Arcones, M. A. (1995b). Weak convergence for the row sums of a triangular array of empirical processes under bracketing conditions. Preprint.

[5] Arcones, M. A. (1996a). The Bahadur-Kiefer representation of U-quantiles. Annals of Statistics 24 $1400-1422$.

[6] Arcones, M. A. (1996b). The Bahadur-Kiefer representation of $L_{p}$ regression estimators. Econometric Theory 12 257-283

[7] Arcones, M. A. and Giné, E. (1995). On the law of the iterated logarithm for canonical U-statistics and processes. Stoch. Proc. Applic. 58 217-245.

[8] Aronszajn, N. (1950). Theory of reproducing kernels. Trans. Amer. Mathem. Soc. 68 337-404.

[9] Bingham, N. H., Goldie, C. M. and Teugels, J. L. (1987). Regular Variation. Cambridge University Press, Cambridge, United Kingdom.

[10] Deheuvels, P. and Mason, D. M. (1990). Nonstandard functional laws of the iterated logarithm for tail empirical and quantile processes. Ann. Probab. 18 1693-1722.

[11] Deheuvels, P. and Mason, D. M. (1991). A tail empirical process approach to some nonstandard laws of the iterated logarithm. J. Theor. Probab. 4 53-85.

[12] Deheuvels, P. and Mason, D. M. (1994). Functional laws of the iterated logarithm for local empirical processes indexed by sets. Ann. Probab. 22 1619-1661.

[13] Deheuvels, P. and Mason, D. M. (1995). Nonstandard local empirical processes indexed by sets. $J$. Statist. Plann. Inference 45 91-112.

[14] Dembo, A. and Zeitouni, O. (1993). Large Deviations Techniques and Applications. Jones and Barlett Publishers, Boston.

[15] Dudley, R. M. (1984). A course on empirical processes. Lect. Notes in Math. 1097 1-142. Springer, New York.

[16] Ellis, R. S. (1984). Large deviations for a general class of random vectors. Ann. Probab. 12 1-12. 
[17] Finkelstein, H. (1971). The law of the iterated logarithm for empirical distributions, Ann. Math. Statist. 42 607-615.

[18] Giné, E. and Zinn, J. (1986). Lectures on the central limit theorem for empirical processes, Lect. Notes in Math. 1221 50-112. Springer-Verlag, New York.

[19] Hall, P. (1981). Laws of the iterated logarithm for nonparametric density estimators. Z. Wahrsch. verw. Gebiete $\mathbf{5 6}$ 47-61.

[20] Hall, P. (1991). On iterated logarithm laws for linear arrays and nonparametric regression estimators. Ann. Probab. 19 740-757.

[21] Härdle, W. (1984). A law of the iterated logarithm for nonparametric regression function estimators. Ann. Probab. 12 624-635.

[22] Kiefer, J. (1972). Iterated logarithm analogues for sample quantiles when $p_{n} \searrow 0$. Proc. Sixth Berkeley Symp. Math. Stat. and Prob. I, 227-244. Univ. of California Press, Berkeley.

[23] Ledoux, M. and Talagrand, M. (1988). Characterization of the law of the iterated logarithm in Banach spaces. Ann. Probab. 16 1242-1264.

[24] Ledoux, M. and Talagrand, M. (1991). Probability in Banach Spaces. Springer, New York.

[25] Parzen, E. (1962). On the estimation of a probability density function and mode. Ann. Mathem. Statist. 33 1065-1076.

[26] Prakasa Rao, B. L. S. (1983). Nonparametric Functional Estimation. Academic Press, New York.

[27] Stout, W. (1974). Almost Sure Convergence. Academic Press, New York. 